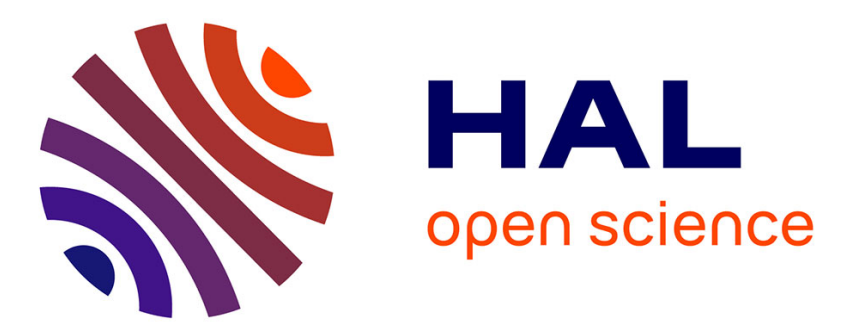

\title{
Control of axisymmetric vortex breakdown in a constricted pipe: Nonlinear steady states and weakly nonlinear asymptotic expansions \\ Philippe Meliga, François Gallaire
}

\section{To cite this version:}

Philippe Meliga, François Gallaire. Control of axisymmetric vortex breakdown in a constricted pipe: Nonlinear steady states and weakly nonlinear asymptotic expansions. Physics of Fluids, 2011, 23, pp.084102. 10.1063/1.3610380 . hal-01067912

\section{HAL Id: hal-01067912 https://hal.science/hal-01067912}

Submitted on 24 Sep 2014

HAL is a multi-disciplinary open access archive for the deposit and dissemination of scientific research documents, whether they are published or not. The documents may come from teaching and research institutions in France or abroad, or from public or private research centers.
L'archive ouverte pluridisciplinaire HAL, est destinée au dépôt et à la diffusion de documents scientifiques de niveau recherche, publiés ou non, émanant des établissements d'enseignement et de recherche français ou étrangers, des laboratoires publics ou privés. 


\title{
Control of axisymmetric vortex breakdown in a constricted pipe: Nonlinear steady states and weakly nonlinear asymptotic expansions
}

\author{
Philippe Meliga and François Gallaire \\ Laboratory of Fluid Mechanics and Instabilities - Ecole Polytechnique Fédérale de Lausanne, \\ CH1015 Lausanne, Switzerland
}

(Received 8 November 2010; accepted 20 June 2011; published online 5 August 2011)

\begin{abstract}
The present paper investigates numerically and theoretically the axisymmetric vortex breakdown occurring in a constricted pipe of infinite extension, i.e., the transition from a smooth columnar state to a breakdown state exhibiting a recirculation bubble. Velocity distributions are prescribed at the pipe inlet under the form of Batchelor vortices with uniform axial velocity and variable levels of confinement. A numerical continuation technique is developed to follow the branches of nonlinear steady solutions when varying the swirl parameter. In the most general case, vortex breakdown occurs abruptly owing to a subcritical, global instability of the non-parallel, viscous columnar solution, and results in the coexistence of multiple stable solutions over a finite range of swirl. For highly confined vortices, a second scenario prevails, where the flow transitions smoothly from the columnar to the breakdown state without any instability. The effect of a low-flow rate jet positioned at the pipe wall is then characterized in the perspective of control. Its effectiveness is evaluated in light of several practically meaningful criteria, namely, the ability of the control to optimize either the stability domain or the topology of the columnar state and its ability to alleviate hysteresis. For each criterion, an optimal jet position is determined from nonlinear simulations, the results being in good agreement with that issuing from an asymptotic expansion of the NavierStokes equations. Finally, we illustrate the importance of physically motivated control strategies by demonstrating how the wall jet technique can be outdone by an appropriate manipulation of the axial velocity profile prescribed at the pipe inlet. (C) 2011 American Institute of Physics.
\end{abstract} [doi:10.1063/1.3610380]

\section{INTRODUCTION}

Vortex breakdown is a widespread phenomenon that affects a variety of flows involving vortices with axial flow, ranging from leading-edge vortices over delta wings to flame holders in combustion devices and atmospheric tornadoes. It consists of an abrupt change in the flow topology when the swirl number $S$, which compares the magnitude of the azimuthal and axial velocity components, exceeds a critical value. The columnar solutions observed at low swirl (i.e., small rotational velocities) are characterized by large axial velocities and negligible axial gradients, whereas the breakdown solutions prevailing at large swirl (i.e., large rotational velocities) exhibit an internal stagnation point. The present study focuses on open swirling jet flows (as opposed to containers with a rotating end wall), where vortex breakdown raises a number of major concerns including hysteretic behaviour and unsteadiness. As an example, the maneuverability of an aircraft is strongly impacted by the existence of multiple stable solutions, whereas the onset of unsteadiness induces structural vibrations responsible for accelerated fatigue.

As reported early from flow visualization studies, vortex breakdown may come in various forms. The basic form is axisymmetric, and the most documented state is that of a steady bubble enclosing a finite region of recirculating fluid, ${ }^{1}$ although Billant $e t$ al. ${ }^{2}$ reported that the vortex may also take the form of an open conical sheet in case the surrounding medium is unbounded. Additional forms can be observed when varying the flow configuration and parameter settings, hence resulting in the existence of complex helical patterns (single, double-helix) with well-defined rotational frequencies. ${ }^{3}$ In return, the understanding of the sequence of events leading to vortex breakdown remains limited despite extensive theoretical, numerical, and experimental researches over the last decades. ${ }^{2,4-8}$ This results in a poor effectiveness of the techniques applied to flow control, ${ }^{9}$ hence motivating the undertaking of further fundamental investigations.

The generally acknowledged idea is that vortex breakdown can be first investigated in terms of a steady axisymmetric model. Secondary helical disturbances may then grow, the mode selection and internal structure of the breakdown pattern being then viewed as a consequence of the stability properties of the axisymmetric breakdown solution itself. ${ }^{7}$ This has been confirmed by the recent numerical simulations of Ruith et al. ${ }^{10}$ who have shown that the early stage of breakdown is axisymmetric. For large swirl numbers, a finite time is needed before this flow pattern is altered by the subsequent development of large-scale spiral waves of various azimuthal wavenumbers, wrapped around and behind the axisymmetric bubble. Moreover, strong evidence has been provided that links the associated, synchronized, helical oscillations to a transition from convective to local absolute instability of wake-like model axisymmetric profiles. ${ }^{10-16}$

Many efforts have also been devoted to the description of the initial axisymmetric breakdown state. ${ }^{17-21}$ Results obtained from various confined and unconfined vortices 
show that only a single stable branch of solutions exists at low Reynolds numbers. When the swirl increases, a smooth vortex breakdown occurs, with a flow pattern changing gradually from the columnar to the breakdown state. In contrast, two distinct stable branches of solutions exist at higher Reynolds numbers. The columnar solution remains stable up to some threshold $S_{\mathrm{b}}$ above which only the disconnected breakdown solution is observed. As the swirl is then decreased, the breakdown state remains stable down to a second threshold $S_{\mathrm{f}} \leq S_{\mathrm{b}}$ below which only the columnar solution is retrieved. Vortex breakdown is then subcritical in the sense of bifurcation theory (not to be confused with the supercritical/subcritical terminology used for inertial waves in parallel vortices with axial flow ${ }^{22}$ ) and characterized by the existence of a hysteresis loop $S_{\mathrm{f}} \leq S \leq S_{\mathrm{b}}$ where the columnar and breakdown states coexist.

The case of a swirling flow in a straight pipe has been considered by Rusak and co-workers. ${ }^{23-25}$ These authors proposed an inviscid mechanism relying on the upstream propagation of disturbance waves in the pipe and on their interaction with the inlet boundary, and further treated the case of weak viscosity and weak variations in the pipe geometry as perturbations from this "ideal" case. ${ }^{26-29}$ Such an approach has provided relevant models consistent with previous and later experimental and numerical results, helping to understand the occurrence of subcritical vortex breakdown (see for instance the recent experiments by Mattner et $a l^{30}$ ). It has also yielded some renewal in the design of control strategies. In particular, Gallaire et al. ${ }^{31}$ successfully quenched the linear development of the Wang and Rusak instability in a finite-length tube by designing a feedback control scheme on a reduced-order system built from the least stable modes.

Still, such results rely on an ideal inviscid columnar state obtained by neglecting the radial velocity of the steady state and by extending to infinity the velocity profiles prevailing at the pipe inlet. We rather consider here the non-parallel columnar solution developing in a circular pipe equipped with a throat. This geometry mimics a realistic experimental set-up (i.e., a convergent annular intake section followed by a slowly divergent test section) and has thus been the subject of several numerical analyses. ${ }^{17-20,32}$ These, however, all rely on a strong assumption of frictionless flow at the pipe wall, meant to ease the numerical implementation when using a streamfunctionvorticity-angular momentum formulation of the governing equations. The present research differs in an important respect as it takes into account the existence of a boundary layer, whose main effect is to delay the onset of vortex breakdown.

Here, we characterize the transition to vortex breakdown over wide ranges of swirl, at a moderate value of the Reynolds number. This may constitute a limitation since many practically related situations involve large scales and thus large Reynolds numbers. Nevertheless, such an approach is relevant in the perspective of control as it allows to rigorously identify the dominant instability mechanisms, which in turn enables to design physically motivated strategies, as will be illustrated by comparing the effect of a low-flow rate jet at the pipe wall issuing from both nonlinear simulations and from asymptotic expansions carried out in the vicinity of the bifurcation threshold. We follow here the line of thought of
Escudier $^{7}$ and view the existence of helical breakdown patterns as the manifestation of secondary instabilities sustained by the axisymmetric breakdown solution. Credit is given to this interpretation by systematical stability analyses showing that all columnar solutions, prior to vortex breakdown, are stable to three-dimensional disturbances. In this geometry, axisymmetric vortex breakdown is, thereby, singled out as a prerequisite to the existence of any other pattern.

The paper is organized as follows: the flow configuration and numerical tools are presented in Secs. II and III. Typical bifurcation diagrams of the nonlinear, uncontrolled solutions are presented in Sec. IV, where we provide evidence that vortex breakdown results from a fold of the non-parallel columnar solution related to a subcritical, global instability. Section $\mathrm{V}$ is devoted to the open-loop control of vortex breakdown by means of an annular jet positioned at the pipe wall. Being physically tractable, such a method is expected to yield potentially many applications and to ease future contact with experimental data. We assess the effect of varying the jet location and flow rate and discuss the success of the control in terms of its impact on the stability and topology of the columnar solution, but also on the ability of the flow to sustain hysteresis. These nonlinear results are then compared to those issuing from a weakly nonlinear asymptotic expansion of the Navier-Stokes equations. Although rigorously valid for small control velocities, the relevance of the asymptotic results will be demonstrated to carry over qualitatively even at realistic parameter settings. In Sec. VI, we focus on an alternative control strategy relying on an appropriate modification of the inlet velocity profile. Finally, the relevance and robustness of the axisymmetric modelisation is discussed in Sec. VII, where we characterize the stability of the various branches of solutions to helical disturbances.

\section{FLOW CONFIGURATION}

In the following, we use standard cylindrical coordinates $r, \theta$, and $z$ with origin taken at the center of the pipe inlet. A sketch of the geometry is presented in Fig. 1: the downstream part of the pipe is an infinite-length, straight pipe of radius $R$, whereas its upstream part of length $L$ exhibits a contraction followed by an expansion whose profile is defined as one period of a cosine function. The wall geometry thus reads

$$
\begin{array}{cl}
r=R(1-\lambda)+\lambda R \cos \left(\frac{2 \pi z}{L}\right), & \forall z \leq L, \\
r=R, & \forall z>L,
\end{array}
$$

where $\lambda$ is the contraction parameter. Physically, the diverging part of the pipe promotes vortex breakdown by creating an adverse pressure gradient, whereas the contracting part in turn prevents the breakdown region from interacting with the computational inlet boundary.

Several numerical analyses ${ }^{17-20,32}$ have been devoted to the characterization of vortex breakdown in similar geometries. All existing results have been obtained under the assumption of frictionless flow at the pipe wall, a somewhat questionable hypothesis for the moderate Reynolds numbers considered, albeit convenient for the streamfunction-vorticity-angular momentum formulation of the Navier-Stokes 


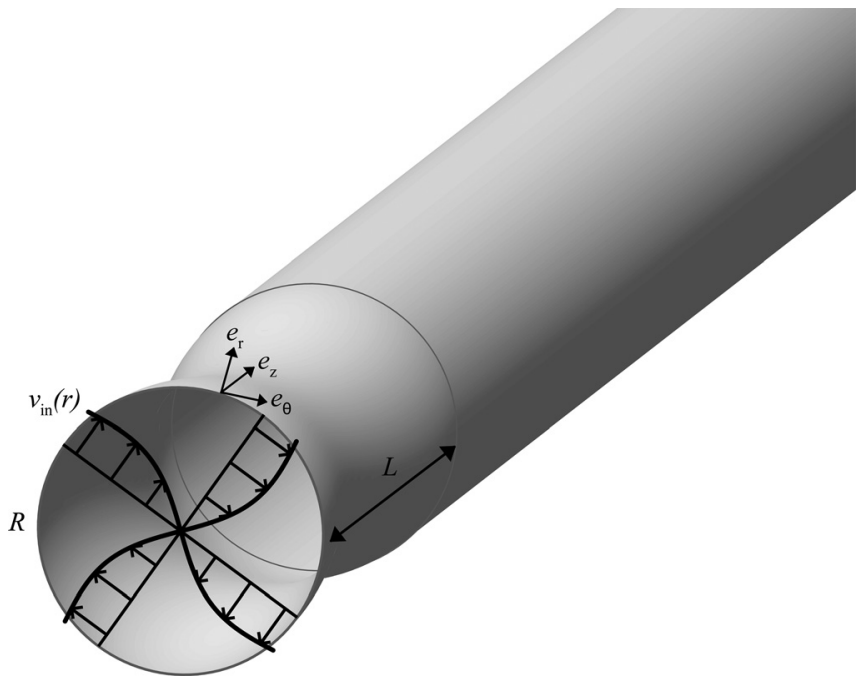

FIG. 1. Three-dimensional sketch of the constricted pipe geometry along with the Batchelor azimuthal velocity distribution defined by Eq. (2).

equations solved in these references. We focus here on settings investigated by Beran and Culick, ${ }^{18}$ Beran, ${ }^{19}$ and Lopez, ${ }^{20}$ namely, $L=5 R$ and $\lambda=0.05$, for which the maximum variation of the pipe radius is $10 \%$ at the contraction throat. Our approach, however, differs in an important respect as the prescribed no-slip boundary condition allows the development of a boundary layer at the pipe wall, whose main effect is to delay the transition to vortex breakdown, as seen later in the paper.

Many of the works aiming at modeling vortex breakdown point out the choice of upstream boundary conditions as a difficult problem. In the present case, the velocity is prescribed at the pipe inlet under the form of a Batchelor vortex with no boundary layer, admitting the dimensional radial, azimuthal, and axial components

$$
\begin{aligned}
& u_{\text {in }}(r)=0, \quad v_{\text {in }}(r)=\Omega_{0} R_{v}^{2} \frac{1-e^{-\left(r / R_{v}\right)^{2}}}{r}, \\
& w_{\text {in }}(r)=w_{\infty}+\left(w_{0}-w_{\infty}\right) e^{-\left(r / R_{v}\right)^{2}}, \quad \forall r \leq R .
\end{aligned}
$$

In the above expressions, $w_{0}$ is the jet centreline axial velocity and $w_{\infty}$ is the jet free-stream velocity that would be reached in the limit $r \rightarrow \infty$, i.e., in the absence of confining wall. $\Omega_{0}$ is the centreline axial vorticity, the complete distribution being a truncated gaussian whose standard deviation $R_{v}$ measures the size of the vortex core. Consequently, we introduce the non-dimensional parameter $h$

$$
h=\frac{R_{v}}{R},
$$

measuring the confinement of the vortex, the two limit-cases $h=O(1)$ and $h \rightarrow 0$ being associated, respectively, with a highly confined vortex and to a vortex in a medium of infinite radial extension. Such profiles can be obtained by means of a honeycomb and have been fitted to several experimental data. ${ }^{33}$ The present configuration may thus provide a realistic model of the flow situation in a pipe as found in a typical experimental apparatus.

The fluid is viscous of dynamic viscosity $\mu$, incompressible, and homogeneous of density $\rho=1$. Its motion is described by a state vector $\boldsymbol{q}=(\boldsymbol{u}, p)^{T}$, with $\boldsymbol{u}=(u, v, w)^{T}$ the three-dimensional velocity field of radial, azimuthal, and axial components $u, v$, and $w$, and $p$ the pressure, solution of the incompressible, axisymmetric Navier-Stokes equations. For consistency with the study of Lopez, ${ }^{20}$ we use the halfradius of the pipe as length scale. It is convenient to use $w_{0}$ as velocity scale, but we normalize the azimuthal component using the secondary velocity scale $R_{v} \Omega_{0}$, which yields the non-dimensional governing equations

$$
\begin{array}{r}
\partial_{r} u+\frac{u}{r}+\partial_{z} w=0 \\
\frac{\partial u}{\partial t}+u \partial_{r} u+w \partial_{z} u-S^{2} \frac{v^{2}}{r}+\partial_{r} p+\partial_{r} \tau_{r r}+\partial_{z} \tau_{r z}+\frac{\tau_{r r}-\tau_{\theta \theta}}{r}=0 \\
\frac{\partial v}{\partial t}+u \partial_{r} v+w \partial_{z} v+\frac{u v}{r}+\partial_{r} \tau_{r \theta}+\partial_{z} \tau_{\theta z}+\frac{2 \tau_{r \theta}}{r}=0 \\
\frac{\partial w}{\partial t}+u \partial_{r} w+w \partial_{z} w+\partial_{z} p+\partial_{r} \tau_{r z}+\partial_{z} \tau_{z z}+\frac{\tau_{r z}}{r}=0
\end{array}
$$

where $\tau_{i j}$ denotes the components of the viscous stress tensor

$$
\tau=\frac{1}{R e}\left(\nabla \boldsymbol{u}+\nabla \boldsymbol{u}^{T}\right) .
$$

Finally, $R e$ and $S$ are the Reynolds and swirl numbers defined by

$$
R e=\frac{\rho R w_{0}}{2 \mu}, \quad S=\frac{R_{v} \Omega_{0}}{w_{0}} .
$$

Unless specified otherwise, we consider here only the case of a plug jet for which $w_{0}=w_{\infty}$. Note that the retained normalization choice has no physical effect but eases the numerical implementation as the non-dimensional inlet boundary condition

$$
\begin{array}{ll}
u_{\text {in }}(r)=0, & v_{\text {in }}(r)=\frac{1-e^{-(r / 2 h)^{2}}}{r / 2 h}, \\
w_{\text {in }}(r)=1, & \forall r \leq 2
\end{array}
$$

is independent of the swirl. 


\section{PROBLEM FORMULATION}

\section{A. Numerical method}

We use the FreeFem ++ software $^{54}$ to generate a twodimensional triangulation of the azimuthal plane $\theta=0$ with the Delaunay-Voronoi algorithm. The computational domain is shown in Fig. 2. $\partial \Sigma_{w}$ is the pipe wall, where we impose the no-slip condition $\boldsymbol{u}=\mathbf{0}$, which represents the key difference with respect to the existing literature. $\partial \Sigma_{\text {in }}$ is the pipe inlet, where we impose the velocity $\boldsymbol{u}=\boldsymbol{u}_{\text {in }}$. Finally, $\partial \Sigma_{\mathrm{a}}$ is the revolution axis, at which the boundary conditions are obtained from mass and momentum conservation as $r \rightarrow 0$, namely, $u=0, \partial_{r} v=0$, and $\partial_{r} w=0$.

In order to apply appropriate outflow conditions, the pipe is first truncated at a finite distance $z=L_{\infty}$. The soobtained computational pipe is then artificially extended down to some distance $z=L_{\infty}+l_{s}$ defining the position of the computational outlet boundary $\partial \Sigma_{\text {out }}$. The domain in between is a sponge region in which the azimuthal component of the velocity is progressively damped to negligible levels (grey shaded area in Fig. 2). In practice, this is achieved by means of artificial dissipation, i.e., the Reynolds number is smoothly decreased from its value defined in Eq. (6) to the small value $R e_{s}=0.1$ at the end of the domain. The Reynolds number in all equations should therefore be replaced by a computational Reynolds number $\widetilde{R e}$ defined by

$$
\begin{aligned}
\widetilde{\operatorname{Re}}(z)=\operatorname{Re} & \text { if } z \leq L_{\infty}, \\
\widetilde{\operatorname{Re}}(z)=\operatorname{Re}+\left(\operatorname{Re} e_{s}-\operatorname{Re}\right) \zeta\left(z, L_{\infty}\right) & \text { if } z \geq L_{\infty},
\end{aligned}
$$

the $\zeta$ function being defined by

$$
\zeta(a, b)=\frac{1}{2}+\frac{1}{2} \tanh \left\{\varsigma \tan \left(-\frac{\pi}{2}+\pi \frac{|a-b|}{l^{s}}\right)\right\},
$$

along with $\varsigma=1.5$. In addition, the numerical dissipation in the sponge region is increased by a progressive grid stretching. Consequently, the fluid reaches the outlet boundary under the form of a Poiseuille flow for which we use the simple free-outflow condition $\left(-p \mathbf{I}+R e_{s}^{-1} \tau\right) \cdot n=0$, with $\boldsymbol{n}$ the outward-pointing vector normal to the domain.

The mesh refinement is controlled by the vertex densities imposed on both external and internal boundaries. All equations are numerically solved by a finite-element method using the same mesh $M_{1}$ built with $L_{\infty}=50$ and $l_{s}=35$, hence resulting in 165300 triangles, the accuracy of the results with respect to the discretization being assessed in Appendix A. A set of equations is first multiplied by $r$ to avoid the singularity on the $r=0$ axis. The associated varia-

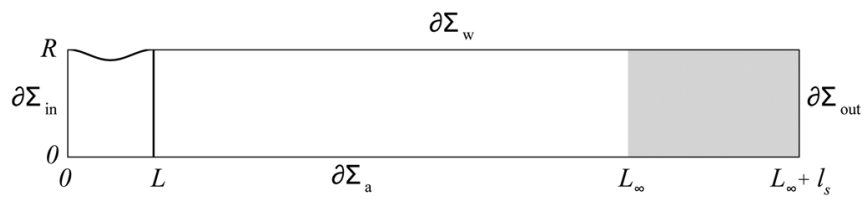

FIG. 2. Schematic of the computational domain: $L$ stands for the length of the contraction, whose geometry is defined by Eq. (1), and $L_{\infty}$ stands for the total length of the computational pipe. The inlet and external boundaries are located at $z=0$ and $z=L_{\infty}+l_{s}$, respectively, where $l_{s}$ is the size of the sponge region used in the numerics, shown as the light grey shaded area. tional formulation is then derived and spatially discretized onto a basis of Arnold-Brezzi-Fortin MINI-elements, ${ }^{35}$ with 3-node $P_{1}$ elements for the pressure (i.e., piecewise linear continuous finite elements with three degrees of freedom at each triangle edge) and $P_{1 \mathrm{~b}}$ elements for each velocity component (same as the $P_{1}$ elements but enriched with a cubic bubble function at the barycenter of each triangle). The sparse matrices resulting from the projection of these variational formulations onto the basis of finite elements are built with the FreeFem ++ software.

\section{B. Nonlinear steady states}

From now on, all governing equations are written as formal relations between differential operators. In particular, Eq. (4) are recast into

$$
\mathcal{B} \frac{\partial \boldsymbol{q}}{\partial t}+\mathcal{M}(\boldsymbol{q}, R e, S)=\mathbf{0},
$$

where $\mathcal{B}$ is a constant-coefficient operator and $\mathcal{M}$ is the axisymmetric nonlinear evolution operator. All calculations are hereafter performed keeping the Reynolds number constant, meaning that steady solutions are equilibrium points in the $(\boldsymbol{q}, S)$ space, hence satisfying

$$
\mathcal{M}(\boldsymbol{q}, S)=\mathbf{0}
$$

In the most general case, branches of solutions are parameterized in terms of the swirl number as $\boldsymbol{q}=\mathcal{F}(S)$ and are computed by means of a classical Newton iterative technique based on the successive iterations of a guess value $\overline{\boldsymbol{q}}{ }^{36} \mathrm{At}$ each step, the increment $\delta \boldsymbol{q}$ is solution of the linear problem

$$
\mathcal{A}(\overline{\boldsymbol{q}}, S) \delta \boldsymbol{q}=-\mathcal{M}(\overline{\boldsymbol{q}}, S),
$$

where $\mathcal{A}=\partial \mathcal{M} / \partial \boldsymbol{q}$ is the axisymmetric linearized evolution operator, the matrix inversion being performed using the sparse direct LU solver implemented in the UMFPACK library. ${ }^{37,38}$ This process is repeated until the $\mathcal{L}^{2}$-norm of the residual of the governing equations becomes smaller than $10^{-12}$.

This method fails in the vicinity of turning points, where several branches coexist at the same swirl. In this case, we use an algorithm similar to that of Beran and Culick, ${ }^{18}$ based on a predictor-corrector technique. ${ }^{39}$ The branches of solutions are parameterized in terms of a pseudo-arclength $l$ as $(\boldsymbol{q}, S)=\mathcal{F}(l)$, the swirl being now part of the unknowns. At each step, the Newton iterations are modified by the addition of a correction term in the direction normal to the vector tangent to the branch of solutions, and the increments $\delta \boldsymbol{q}$ and $\delta S$ are sought as the solutions of the modified problem

$$
\begin{aligned}
& \mathcal{A}(\overline{\boldsymbol{q}}, S) \delta \boldsymbol{q}+\frac{\partial \mathcal{M}}{\partial S}(\overline{\boldsymbol{q}}, S) \delta S=-\mathcal{M}(\overline{\boldsymbol{q}}, S), \\
& \mathcal{T}_{\boldsymbol{q}}(\overline{\boldsymbol{q}}, S) \cdot \delta \boldsymbol{q}+\mathcal{T}_{S}(\overline{\boldsymbol{q}}, S) \cdot \delta S=0
\end{aligned}
$$

where $\mathcal{T}_{q}$ and $\mathcal{T}_{S}$ are the flow and swirl components of the tangent vector, and $\cdot$ refers to the canonical hermitian scalar product in $\mathbb{C}^{n}$. 


\section{Linear stability analysis}

The stability of the steady solutions is systematically analyzed by means of eigenvalue calculations. To this end, we have extended the solver presented in Ref. 34. in order to take into account the existence of a non-zero steady azimuthal velocity. Axisymmetric disturbances are sought under the form of normal modes of growth rate $\sigma$ and pulsation $\omega$ according to

$$
\boldsymbol{q}^{\prime}=\hat{\boldsymbol{q}}(r, z) e^{(\sigma+\mathrm{i} \omega) t}+\text { c.c. },
$$

where $\hat{\boldsymbol{q}}=(\hat{\boldsymbol{u}}, \hat{p})^{T}$ is the so-called global mode and c.c. denotes the complex conjugate of the preceding expression. Introducing $\boldsymbol{q}+\epsilon \boldsymbol{q}^{\prime}$ into the governing equations and retaining only terms of order $\epsilon$ yields a system of linear equations governing the normal mode under the form of the generalized eigenvalue problem

$$
(\sigma+\mathrm{i} \omega) \mathcal{B} \hat{\boldsymbol{q}}+\mathcal{A}(\boldsymbol{q}, S) \hat{\boldsymbol{q}}=\mathbf{0},
$$

to be solved with homogeneous boundary conditions linearized from the Navier-Stokes conditions defined in Sec. III A. To this end, we use the "Implicitly Restarted Arnoldi method" of the ARPACK library based upon a shift and invert strategy. ${ }^{40}$ To normalize the global modes, we impose first the phase of the radial velocity to be zero at $r=0.5$ and $z=5$, i.e., $\hat{u}(0.5,5)$ is real positive. The eigenmode energy is then normalized to unity in the computational domain.

\section{Methodology}

We use the minimum of the axial velocity $w_{\min }$ in order to monitor the development of the solutions when varying the swirl. The latter is defined as

$$
w_{\min }=\min \left\{w \mid r \leq R_{v}, z \leq L_{\infty}\right\} \equiv w\left(r_{\min }, z_{\min }\right),
$$

meaning that we exclude the sponge region for physical relevance, as well as the boundary layer region, since the axial velocity drops to zero at the pipe wall. $w_{\min }$ can therefore be viewed as the minimum velocity that would be estimated from a fictitious parallel, inviscid vortex core obtained by extending to infinity the inlet velocity profiles. The soobtained bifurcation diagrams evidence the existence of distinct branches of solutions corresponding to columnar states $\left(w_{\min }>0\right)$ and breakdown states $\left(w_{\min }<0\right)$, as well as intermediate states referred to as being decelerated.

In order to clarify the discussion, we use the following terminology:

- $S_{\mathrm{b}}$ is the swirl at the first backward turning point, if any.

$-S_{\mathrm{f}}$ is the swirl at the first forward turning point, if any.

- $S_{0}$ is the swirl for which the minimum velocity $w_{\min }$ first comes to zero.

$-S_{\mathrm{d}}$ is the swirl above which the flow starts to decelerate even though the solution may remain columnar up to a larger value. For the present plug axial velocity profiles, the latter can be defined as

$$
S_{\mathrm{d}}=\max \left\{S \mid w_{\min }=1\right\} .
$$

By construction, no deceleration occurs in the range $S \leq S_{\mathrm{d}}$, where the solution is said to be fully columnar. We also define $S_{1}$ (respectively $S_{2}$ ) as the critical swirl above which no columnar solution exists (respectively the critical value below which no breakdown solution exists). Since vortex breakdown can occur either through a subcritical or a smooth transition, these critical values are obtained straightforwardly as

$$
\begin{array}{cc}
\text { subcritical vortex breakdown : } & \left\{\begin{array}{l}
S_{1}=S_{\mathrm{b}} \\
S_{2}=S_{\mathrm{f}},
\end{array}\right. \\
\text { smooth vortex breakdown : } & S_{1}=S_{2}=S_{0}
\end{array}
$$

\section{AXISYMMETRIC VORTEX BREAKDOWN OF THE UNCONTROLLED FLOW}

\section{A. Nominal configuration}

We consider the nominal configuration defined by $h=1 / 2$, i.e., the radius of the external pipe is twice that of the vortex core. The bifurcation diagram obtained for $R e=500$ is presented in Fig. 3: 186 solutions have first been computed using the method presented in Sec. III B, $w_{\min }$ being extracted from each of these and the solid line being plotted without further interpolation. This allows to identify three branches of solutions connected via turning points at the swirl numbers $S_{\mathrm{b}}=1.893$ and $S_{\mathrm{f}}=1.822$ (symbols labeled (2) and (4) in Fig. 3, respectively).

The first branch prevailing in the range $S \leq S_{\mathrm{b}}$ (upper thick black line in Fig. 3) exhibits large, positive values of $w_{\min }$ and smooth streamline patterns characteristic of the columnar state (see Fig. 4(a) for the swirl $S=1.75$ labeled (1) in Fig. 3). The solution remains fully columnar up to the swirl $S_{\mathrm{d}}=1.852$ indicated by the triangle symbol. It then starts to decelerate, the relative decrease in $w_{\min }$ being already $50 \%$ for $S=S_{\mathrm{b}}$. Consistently, the flow pattern shown in Fig. 4(b) points out the existence of significant axial velocity gradients along with a low-velocity region downstream of the contraction outlet, as an indicator of the initial onset of vortex breakdown.

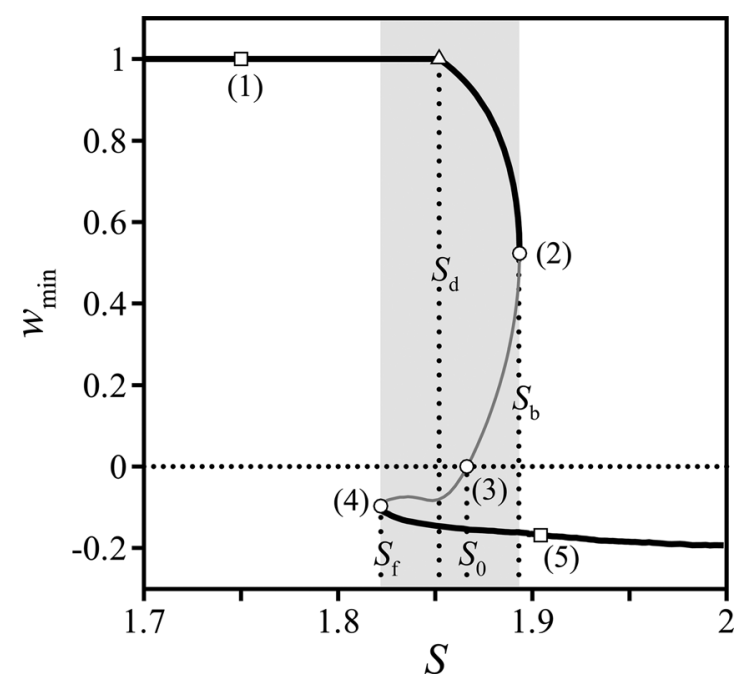

FIG. 3. Bifurcation diagram illustrating the onset of vortex breakdown as the swirl is increased. The minimum axial velocity $w_{\min }$ is plotted as a function of the swirl- $R e=500$. 


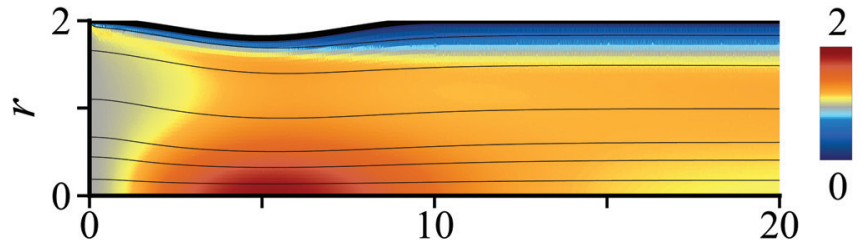

(a)

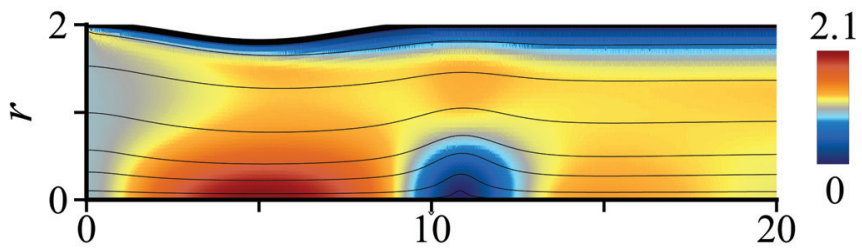

(c)

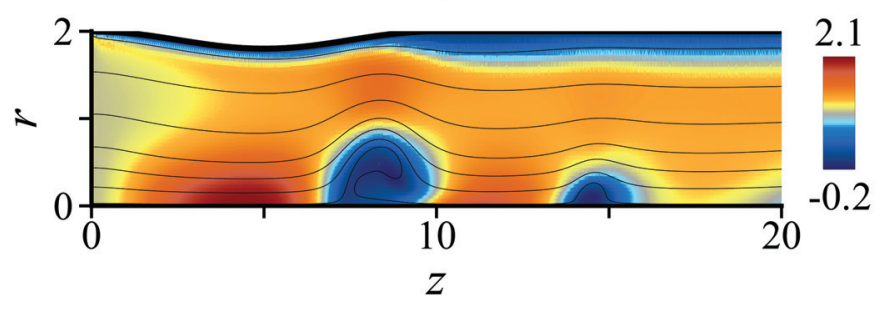

(e)

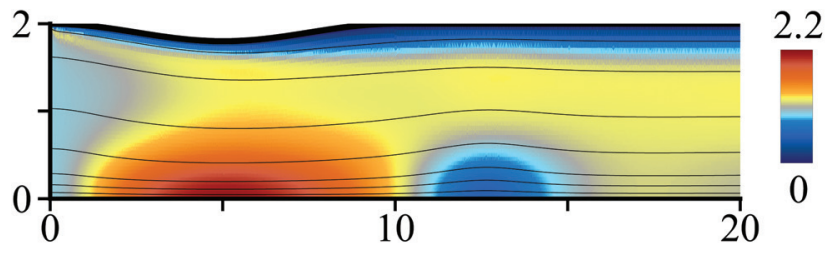

(b)

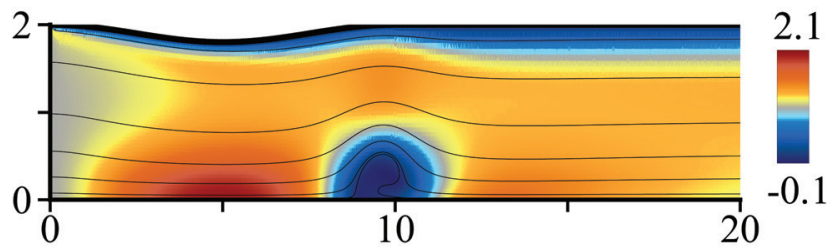

(d)

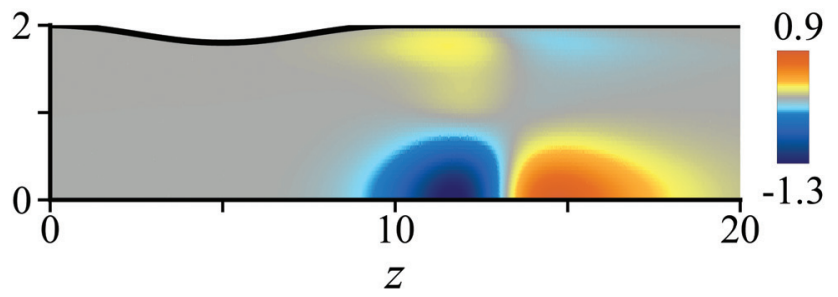

(f)

FIG. 4. (Color) (a)-(e) Spatial distribution of axial velocity and characteristic streamline patterns for the steady solutions corresponding to the swirl numbers labeled from (1) to (5) in Fig. $3-R e=500$. (f) Spatial distribution of axial velocity for the breakdown global mode at threshold of instability $-S=1.893, R e=500$.

The results of the stability analysis are reported in Fig. $5(\mathrm{a})$, where we present the evolution of the axisymmetric eigenvalues as the swirl varies. In practice, a spectrum has been computed for each computational point along the bifurcation curve, and only the growth rates of the least stable eigenvalues are reported ultimately. Although the columnar solution remains stable, with all eigenvalues lying in the $\sigma \leq 0$ halfplane, there exists a branch of steady modes shown as the circle symbols, whose growth rate continuously increases with the swirl until it becomes marginally stable at $S=S_{\mathrm{b}}$. The backward turning point encountered in the bifurcation diagram therefore coincides with the destabilization of this global mode of eigenvector $\hat{\boldsymbol{q}}$ that will be from now on denoted the breakdown mode. Figure 5(f) shows the spatial structure of the axial velocity $\hat{w}$ at threshold, which is dominated by large magnitudes downstream of the contraction, i.e., precisely in the lowvelocity region visible in Fig. 4(b).

A second branch exists in the range $S_{\mathrm{f}}<S<S_{\mathrm{b}}$ (fine grey line in Fig. 3 ) for which $w_{\text {min }}$ gradually decreases until it becomes zero for the swirl $S_{0}=1.866$ labeled (3) in Fig. 4. At this point, the flow is on the verge of recirculating, as illustrated by the distorted streamlines shown in Fig. 4(c). The eigenvalues shown in Fig. 5(b) indicate that these decelerated states are unstable to the breakdown mode, meaning that they are never observed in practice. The growth rate of the breakdown mode reaches a maximum for $S=1.857$, i.e., close to the value $S_{0}$ at which the recirculating bubble sets in, consistently with the intuition that a small but finite amount of disturbances is required for breakdown to occur. The growth rate then continuously decreases until the breakdown mode becomes marginally stable again for $S=S_{\mathrm{f}}$, i.e., at the forward turning point.
The third branch prevailing in the range $S \geq S_{\mathrm{f}}$ (lower thick black line in Fig. 3) exhibits negative values of $w_{\min }$ characterizing the breakdown state, and the streamline patterns now reveal the existence of a fully developed bubble downstream of the contraction. Note that only a single bubble is found in the vicinity of the threshold value $S_{\mathrm{f}}$, as illustrated in Fig. 4(d), but that a series of subsequent bubbles develop at larger swirls (see Fig. 4(e) for the value $S=1.904$ labeled (5) in Fig. 3). The eigenvalues in Fig. 5(c) indicate that this solution is stable to axisymmetric disturbances. The growth rate of the dominant mode first keeps decreasing, whereas a series of weakly stable branches set in, whose growth rates tend to oscillate as the swirl increases. Even though they remain stable, these modes may be responsible for the generation of the secondary bubbles through their nonlinear interaction with the breakdown mode as their spatial structures consist in positive and negative velocity perturbations alternating downstream of the contraction in finite number (not shown here for conciseness). Note also that the breakdown solution is only slightly stable for $S \sim S_{\mathrm{b}}$. Depending on the sensitivity of this specific eigenvalue to a modification of the geometrical and flow settings, it may thus happen that a secondary instability sets in at this stage.

These results are fully consistent with a subcritical vortex breakdown occurring at $S_{1}=S_{\mathrm{b}}$, the range of hysteresis $\Delta S=S_{1}-S_{2}$ being indicated by the light grey shaded area in Fig. 3. They also demonstrate that its onset can be interpreted as the consequence of a fold of the non-parallel columnar solution related to the global instability of the breakdown mode. Note that the critical value can be compared to that previously reported for the same Reynolds number and same confinement parameter, but for frictionless 

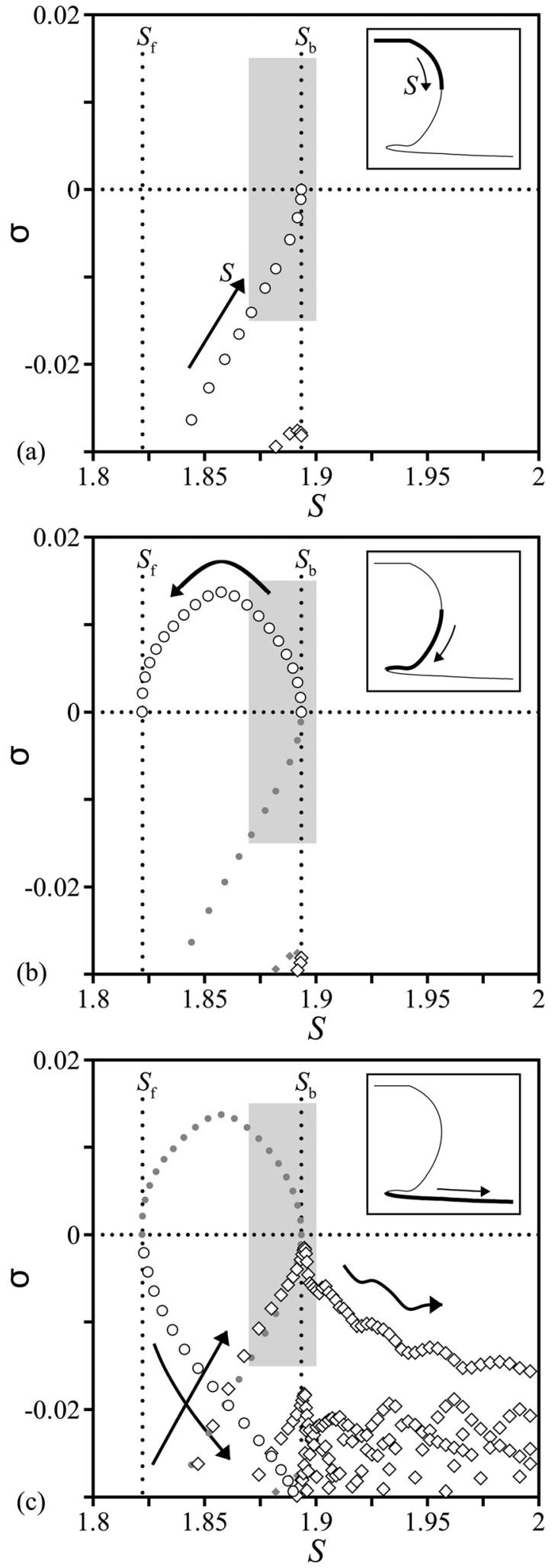

FIG. 5. Stability of the steady solutions presented in Fig. $3-R e=500$. (a) Growth rate of the axisymmetric disturbances, computed as the swirl number varies along the bifurcation curve of the columnar solution $\left(S \leq S_{\mathrm{b}}\right)$. The direction of variation of the swirl number is indicated by the black arrows. (b) Same as (a) for the decelerated states $\left(S_{\mathrm{f}}<S<S_{\mathrm{b}}\right)$. (c) Same as (b) for the breakdown solution $\left(S \geq S_{\mathrm{f}}\right)$. The light grey shaded area indicates the domain for which the growth rate of the breakdown mode is reported in Fig. 16.

flow at the pipe wall, namely, $S_{1} \sim 1.480 .{ }^{18-20}$ One sees that the presence of the boundary layer significantly postpones the onset of vortex breakdown as the critical swirl is increased by approximately $20 \%$. A possible explanation is that the boundary layer artificially reduces the pipe section perceived by the vortex and therefore increases its confinement, a point discussed in Sec. IV B.

\section{B. Effect of confinement}

In order to assess the robustness of the subcritical scenario, similar calculations have been carried out by varying the confinement parameter in the range $1 / 5 \leq h \leq 1 / 1.2$. In practice, we have mimicked the flow situation relevant to an experimental apparatus by varying the size of the vortex and keeping the pipe geometry constant although this choice makes the numerics more demanding at small confinement parameters. We have also rescaled the Reynolds number defined from Eq. (6) in order to keep constant its counterpart based on the vortex core radius $R_{v}$, which ensures that the viscous phenomena at work at the scale of the vortex core are comparable from one simulation to the other. The effect of confinement on the azimuthal velocity distribution imposed in the inlet section is illustrated in Fig. 6, the profile associated with a gaussian vorticity distribution being retrieved in the limit $h \rightarrow 0$.

The results are qualitatively similar to those documented for the nominal configuration. In particular, vortex breakdown is systematically subcritical, except in the most confined case $(h=1 / 1.2)$ which yields a smooth transition. The critical swirl $S_{1}=S_{\mathrm{b}}(h)$ has been computed as a function of the confinement parameter: results are shown in Fig. 7(a), where each open circle marks a computational data point issuing from the derivation of a complete bifurcation diagram identical to that shown in Fig. 3. We find that the critical swirl increases with $h$, i.e., a high level of confinement delays the onset of vortex breakdown. On the one hand, these results are consistent with the physical intuition that creating a recirculating bubble is more intricate in a small-radius pipe since the fluid willing to recirculate must oppose the presence of a solid wall. On the other hand, they support the interpretation of the effect of viscous friction at the pipe wall in terms of a modification of the vortex confinement.

The solid line in Fig. 7(a), which stands for the linear regression line fitting the exact numerical values, evidences the existence of a simple affine relation

$$
S_{1}=S_{1 \infty}+K h
$$

with $K \sim 1.439$ and $S_{1 \infty} \sim 1.187$. Note that the limit case $h \rightarrow 0$ is not exactly that of a free-vortex. It has been indeed checked with the numerics that the presence of the contraction induces pressure gradients at the pipe wall, even in the least confined case, whereas pressure should not vary at far distances in the case of a free vortex. This means that the value 1.187 does not represent the swirl at which breakdown would occur in the absence of confining wall, which can issue only from specific computations carried out with appropriate far field conditions. ${ }^{41}$

Finally, we propose to rescale all results in terms of the new swirl

$$
\tilde{S}=\frac{1}{h}\left(S-S_{1 \infty}\right),
$$




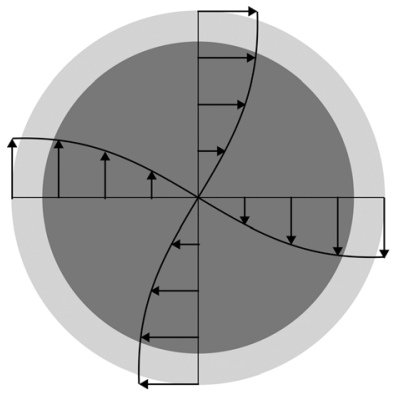

(a)

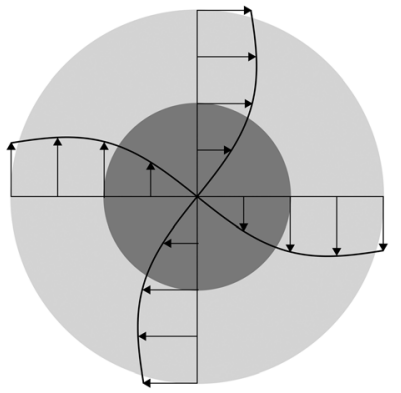

(b)

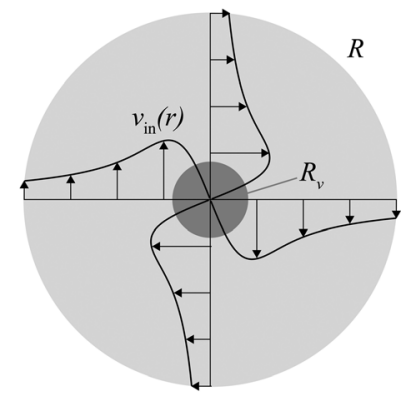

(c)

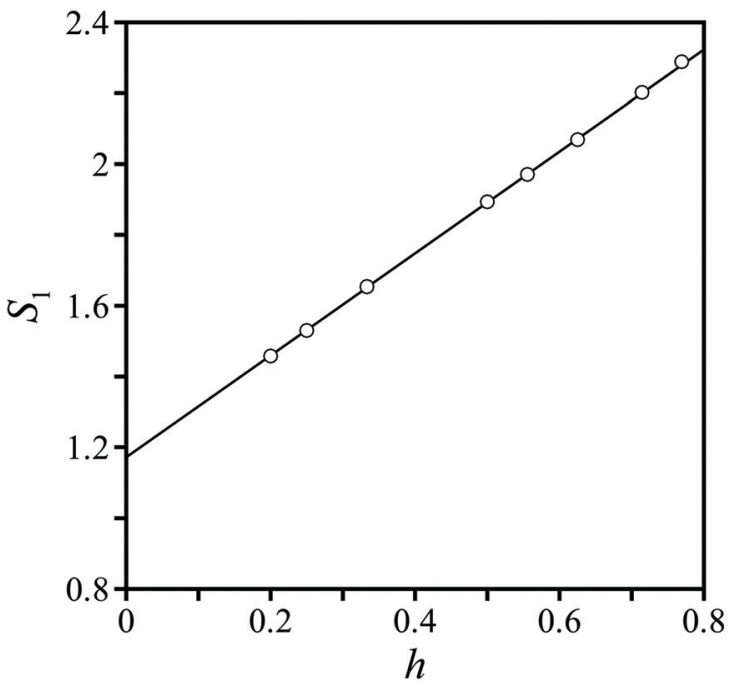

(a)

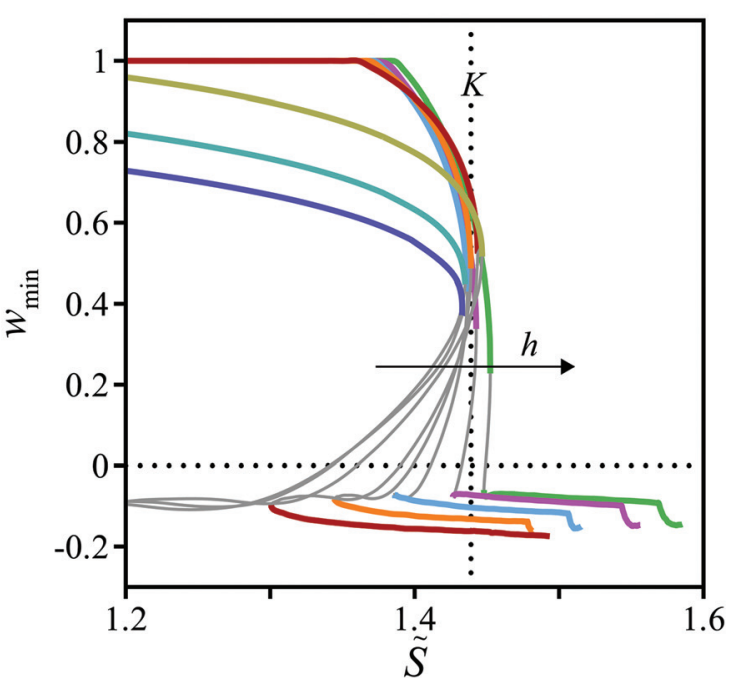

(b)

FIG. 6. Distributions of azimuthal velocity imposed at the pipe inlet for various confinement parameters. The light and dark shaded areas correspond to the pipe and vortex sections, respectively. (a) $h=1 / 5$ (least confined case). (b) $h=1 / 2$ (nominal case). (c) $h=1 / 1.2$ (most confined case).

FIG. 7. (Color) Effect of confinement on the onset of vortex breakdown. (a) Critical swirl $S_{1}$ computed for eight different confinement parameters in the range $1 / 5 \leq h \leq 1 / 1.3$. The circle symbols and solid line stand respectively for the computational data points and for the results issuing from a linear regression fit. (b) Bifurcation diagrams computed for the same values of $h$, rescaled in terms of the swirl $\tilde{S}$ defined by Eq. (20). The vertical dotted line marks the value $\tilde{S}=K=1.439$.

which can be viewed as a modified swirl built from the centreline axial vorticity and from the pipe radius $R$. These reference scales are appropriate for the present configuration as they allow to collapse remarkably all bifurcations thresholds close to the intrinsic value $\tilde{S}_{1}=K$, as illustrated in Fig. 7(b).

\section{CONTROL BY A JET AT THE PIPE WALL}

For clarity, all quantities of interest pertaining to the uncontrolled flow are from now denoted by an ini superscript. In the perspective of control, we focus on the only nominal configuration (i.e., the confinement parameter is set to $h=1 / 2$ ) and investigate how vortex breakdown can be affected by a small jet of gaussian velocity localized at the pipe wall. The jet is set at an axial position $z_{\mathrm{j}}$ measuring the distance from the jet center to the pipe inlet (Fig. 8) and defining the so-called injection section. We consider only the case of an injection normal to the wall (this choice being justified in the following) and define the jet velocity accordingly as

$$
\boldsymbol{u}_{\mathrm{c}}=u_{\mathrm{c}}^{\perp} \boldsymbol{n},
$$

the control velocity being imposed in the numerics through the boundary condition $\boldsymbol{u}=\boldsymbol{u}_{\mathrm{c}}$ along $\partial \Sigma_{\mathrm{w}}$. The width of the jet is $5 \%$ of the pipe radius, as measured by the standard deviation of the gaussian distribution, and the profile is normalized so as to prescribe the relative amplitude with respect to the total flow rate injected within the pipe, i.e.,

$$
\eta=\int_{\partial \Sigma_{\mathrm{w}}} u_{\mathrm{c}}^{\perp} r \mathrm{~d} l / \int_{\partial \Sigma_{\mathrm{in}}} w r \mathrm{~d} r
$$

with $\mathrm{d} l$ the length element along $\partial \Sigma_{\mathrm{w}}$.

\section{A. Effect of the jet position}

We assess first the effect of varying the position of the jet at a constant flow rate representing 3\% of the inlet flow rate, i.e., $\eta=3 \%$. Thirty-two distinct positions have been considered in the range $1 \leq z_{\mathrm{j}} \leq 25$, the resolution between two computational cases varying from $\Delta z_{\mathrm{j}}=0.1$ (close to the contraction outlet) to $\Delta z_{\mathrm{j}}=4$ (far downstream). Owing to the numerous detrimental consequences of vortex breakdown, the success and effectiveness of a given control strategy can be evaluated in light of several criteria or targets:

- ability of the control to restore the existence of the columnar solution,

- ability to alleviate hysteresis, 


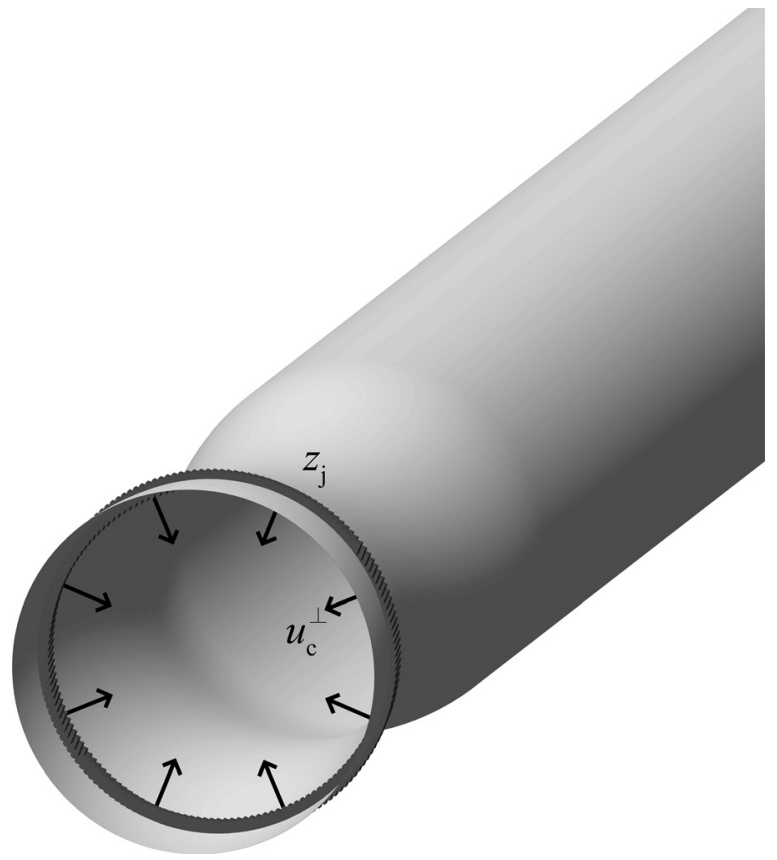

FIG. 8. Three-dimensional sketch of the pipe equipped with an annular jet within the contraction.

- ability to optimize the topology of the columnar solution, the practical relevance of each criterion being discussed in the incoming sections.

\section{Restoring the existence of the columnar solution}

A first control target can be to extend the domain of existence of the columnar solution. The effect of the control can be quantified in terms of the critical swirl $S_{1}$ at which vortex breakdown occurs. The latter is expected to reach a maximum $S_{1, \mathrm{opt}}$ for a jet position $z_{\mathrm{j}, \text { opt }}$ referred to as being optimal, such that

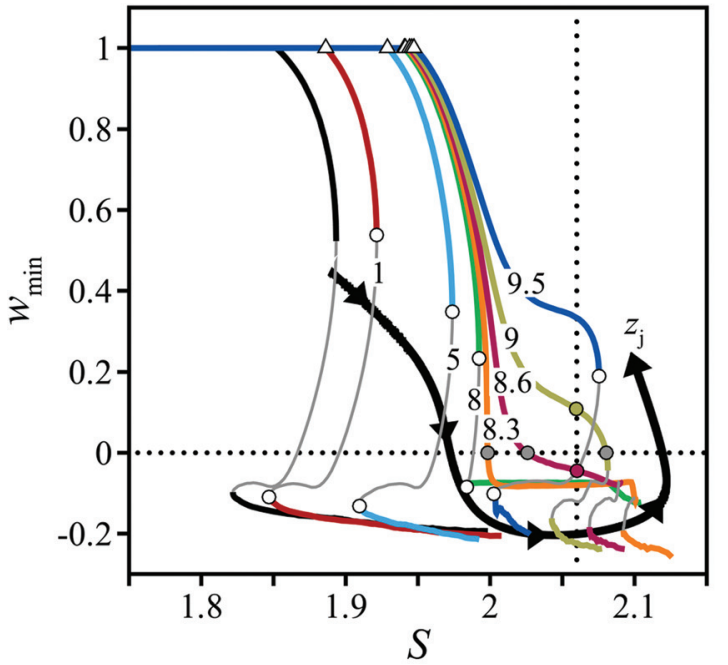

(a)

$$
S_{1, \mathrm{opt}}=\max _{z_{\mathrm{j}}}\left\{S_{1}\right\} \equiv S_{1}\left(z_{\mathrm{j}, \mathrm{opt}}\right)
$$

and whose value is to be determined. To this end, we carry out nonlinear steady state computations and build a bifurcation diagram for each value of $z_{\mathrm{j}}$. We identify then vortex breakdown as being subcritical or smooth and determine eventually the value of $S_{1}$. The controlled columnar states have been checked to be stable to both steady and unsteady axisymmetric disturbances. In contrast, the stability of the breakdown states has been assessed by restricting to steady disturbances only, as the possible growth of disturbances responsible for the subsequent onset of unsteadiness lies out of the scope of the study. Results are synthesized in Figs. 9(a) and 9(b), where we present the diagrams obtained for representative positions inside and outside the contraction $\left(z_{\mathrm{j}} \leq 10\right.$ and $z_{\mathrm{j}} \geq 10$, respectively). Stable (respectively unstable) branches of solutions are shown as the thick coloured lines (respectively the fine grey lines), the branches of solutions pertaining to the uncontrolled configuration being reported for comparison as the leftmost black lines.

The results obtained when displacing the jet downstream from the pipe inlet can be summarized as follows:

- For $z_{\mathrm{j}} \leq 8.2$, vortex breakdown remains subcritical and the critical swirl $S_{1}=S_{\mathrm{b}}\left(z_{\mathrm{j}}\right)$ increases with $z_{\mathrm{j}}$ (rightmost open circle symbols in Fig. 9(a)), meaning that the control gains effectiveness.

- For $8.3 \leq z_{\mathrm{j}} \leq 9$, vortex breakdown is smooth and the critical value $S_{1}=S_{0}\left(z_{\mathrm{j}}\right)$ increases rapidly with $z_{\mathrm{j}}$ (filled circle symbols), meaning that the control keeps being more and more effective. Another interesting result is the existence of a backward turning point $S_{\mathrm{b}}\left(z_{\mathrm{j}}\right)$ in the $w_{\min } \leq 0$ halfplane at which the breakdown solution ultimately becomes unstable to axisymmetric disturbances.

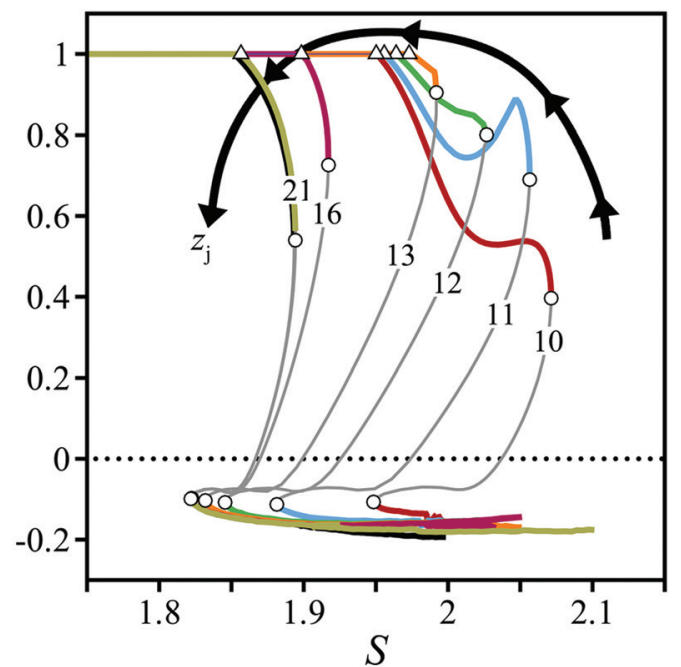

(b)

FIG. 9. (Color) Bifurcation diagrams obtained by varying the position of a control jet along the pipe wall, the distance from the pipe inlet being indicated by the superimposed labels $-\eta=3 \%, R e=500$. (a) Jet inside the contraction $\left(z_{\mathrm{j}} \leq 10\right)$. (b) Jet outside the contraction $\left(z_{\mathrm{j}} \geq 10\right)$. Stable (respectively unstable) branches of solutions are shown as the thick coloured lines (respectively the fine grey lines). The branches of solutions pertaining to the uncontrolled flow are reported for comparison as the leftmost black lines. For each jet position, the critical values $\mathrm{S}_{1}$ and $\mathrm{S}_{2}$ are indicated by either a pair of open circles (subcritical vortex breakdown) or a single filled circle (smooth vortex breakdown). Finally, the triangles indicate the swirl $S_{\mathrm{d}}$ above which the fully columnar solution ceases to exist. In order to ease the reading, the evolution of the critical swirl with the jet position is enhanced by the thick black arrows. The vertical line in (a) marks the value $S=2.06$, for which the steady solutions obtained at $z_{\mathrm{j}}=8.6$ and $z_{\mathrm{j}}=9$ (coloured symbols) are shown in Fig. 13. 
- For $9.5 \leq z_{\mathrm{j}} \leq 12$, the subcritical breakdown scenario is recovered (open circle symbols in Fig. 9) as the backward turning point previously identified has now migrated into the $w_{\min } \geq 0$ half-plane. Note the complex structure of the bifurcation curve as the columnar state successively decelerates, accelerates, decelerates more abruptly and ultimately breaks down at a critical swirl $S_{1}=S_{\mathrm{b}}\left(z_{\mathrm{j}}\right)$. The latter value decreases slowly as $z_{\mathrm{j}}$ increases, meaning that the control becomes less effective.

- For $z_{\mathrm{j}} \geq 13$, vortex breakdown remains subcritical, but the bifurcation curves are now reminiscent of that of the uncontrolled configuration, i.e., the flow does not accelerate anymore. The critical swirl keeps decreasing until the effect of the jet becomes negligible for $z_{\mathrm{j}} \gtrsim 20$.

The effect of the control is best illustrated in Fig. 10, where we report the evolution of the critical swirl as a function of the jet position. Here, each symbol indicates a computational data point whereas the solid curve is the connecting line obtained by spline interpolation. Confirmation comes that the effect of the jet remains limited if located too close from the pipe inlet or too far downstream. In contrast, a high effectiveness is achieved by positioning the jet in the vicinity of the contraction outlet. The maximum value $S_{1, \mathrm{opt}}=2.08$ is reached for the optimal position $z_{\mathrm{j} \text {,opt }} \sim 9$, which represents an increase by $10 \%$ with respect to the value achieved when no control is applied.

At this stage, it remains very intricate to establish the exact mechanism by which the wall normal jet acts. We have mentioned in Sec. IV that our results support the interpretation of vortex breakdown in terms of a linear, global instability. From this point of view, the jet constitutes a forcing term affecting the stability of the columnar solution. Recent studies have considered the effect of open-loop control on flows dominated by instability modes beating at well-defined frequencies. The results can be synthesized as follows: if steady, the control mainly acts at the base flow level, namely, it modi-

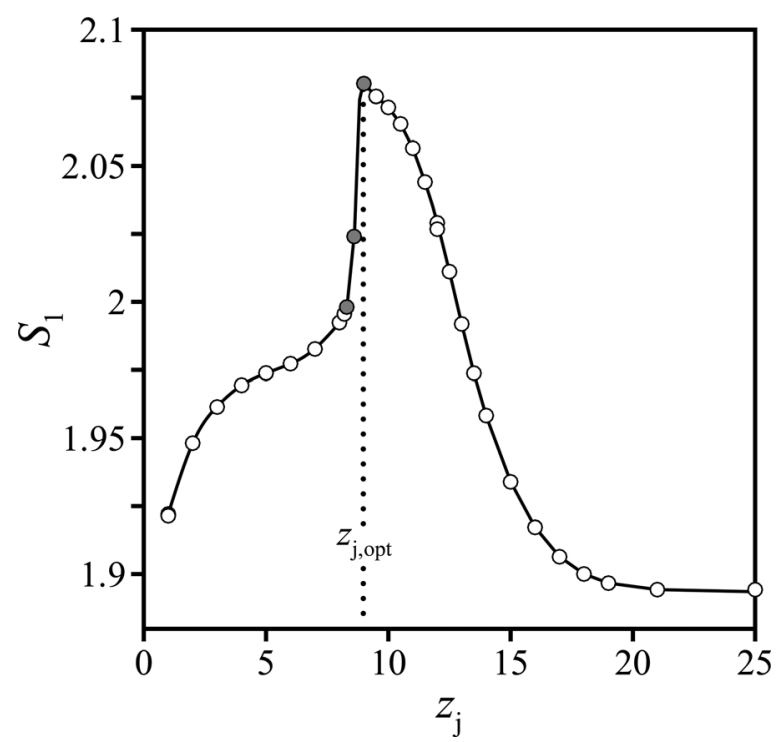

FIG. 10. Critical swirl $S_{1}$ computed as a function of the jet position $z_{\mathrm{j}}$. The position for which $S_{1}$ is maximum defines the so-called optimal jet position $z_{\mathrm{j}, \mathrm{opt}}$ for a control aiming at restoring the existence of the columnar solution- $\eta=3 \%, \operatorname{Re}=500$. fies the base flow on which disturbances develop, ${ }^{4-47}$ which impacts in return the linear stability problem. If harmonic, the control mainly acts at the perturbation level, the idea being to quench the instability by enforcing resonance. ${ }^{48,49}$ Such interpretations are rigorously valid in the limit of small control amplitudes. For larger amplitudes, the linear development of disturbances is similarly affected, but this effect is either enhanced ${ }^{45}$ or limited ${ }^{47}$ by nonlinearities. Nevertheless, in the flow studied herein, both the columnar solution and the breakdown mode are steady and axisymmetric. In consequence, it is impossible to discriminate whether the jet acts at the level of either one or the other or at both levels simultaneously.

Moreover, getting a clear picture of the mechanism at work from an examination of the nonlinear steady states is uneasy. On the one hand, the jet increases the mean flow rate injected within the pipe, but this effect is in a way trivial since it corresponds to a simple rescaling of the swirl, hence contrasting with the significantly distorted bifurcation diagrams displayed in Fig. 9. On the other hand, the controlled and uncontrolled columnar solutions cannot be compared one with the other, for there exist precisely no uncontrolled solution at swirls for which the effect of the control is significant.

In the following, we propose to focus on the axial position $z_{\min }$ defined from Eq. (16) as a mean to monitor the evolution of the low-velocity region ultimately degenerating into the primary recirculating bubble. Results pertaining to the uncontrolled flow are shown in Fig. 11(a). The thick line reproduces the bifurcation curve shown in Fig. 3, the presentation being restricted to the only columnar branch in order to ease the discussion. Note that $z_{\min }$ is uniquely defined only in the range $S>S_{\mathrm{d} \text {,ini }}$, where $S_{\mathrm{d} \text {,ini }}$ is the swirl above which the uncontrolled flow starts to decelerate, defined in Sec. III $\mathrm{D}$, here indicated by the triangle symbol. One sees that the low-velocity region develops initially far downstream $\left(z_{\min } \sim 16\right)$. When the swirl increases, it grows in space and propagates upstream until it finally breaks down slightly downstream the contraction outlet $\left(z_{\min } \gtrsim 12\right.$ /square symbol) when subjected to the adverse pressure gradient. A similar scenario is thus likely to prevail if the jet is positioned sufficiently far upstream of the contraction outlet. In this case, the jet only acts through the increase in the total flow rate, hence explaining that the bifurcation diagrams undergo a simple shift with no distortion in Fig. 9(a). If the jet is positioned sufficiently far downstream, then the low-velocity region starts developing upstream from the injection section, so that no effect should be expected, as observed in Fig. 9(b).

The jet may thus affect vortex breakdown in a non-trivial way only if the injection section departs moderately from the contraction outlet. Confirmation comes from Fig. 11(b) where we report the evolution of the position $z_{\min }$ computed for a jet position $z_{\mathrm{j}}=11$. The bifurcation curve of the columnar solution is reported from Fig. 9(b) as the thick light blue curve. The jet is seen to yield a significant effect when the low-velocity regions is located at a small but finite distance downstream of the injection section, namely, when it moves upstream from $z_{\min } \sim 14.9$ to $z_{\min } \sim 12.6$ (grey shaded area whose lower and upper bounds are indicated by the leftmost square symbols). In this case, the jet is able to confine the 


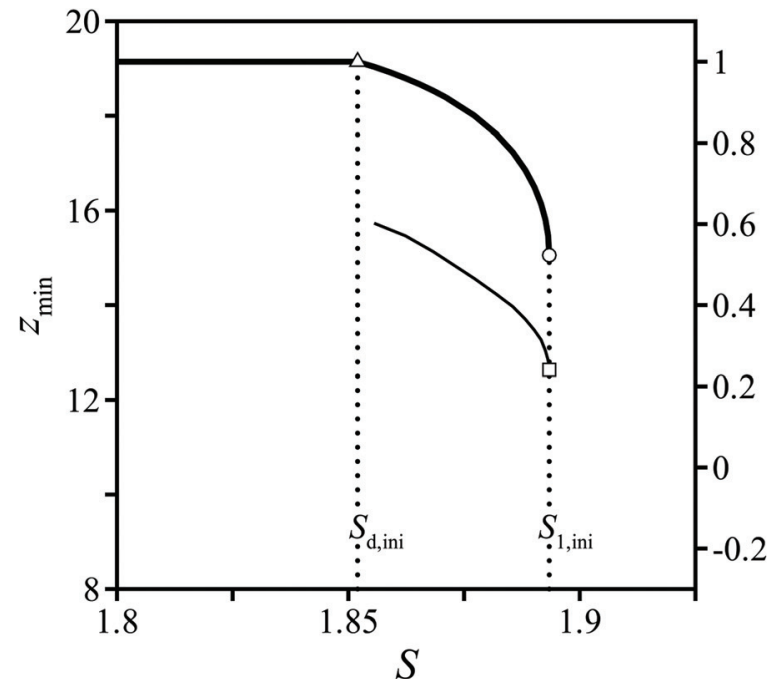

(a)

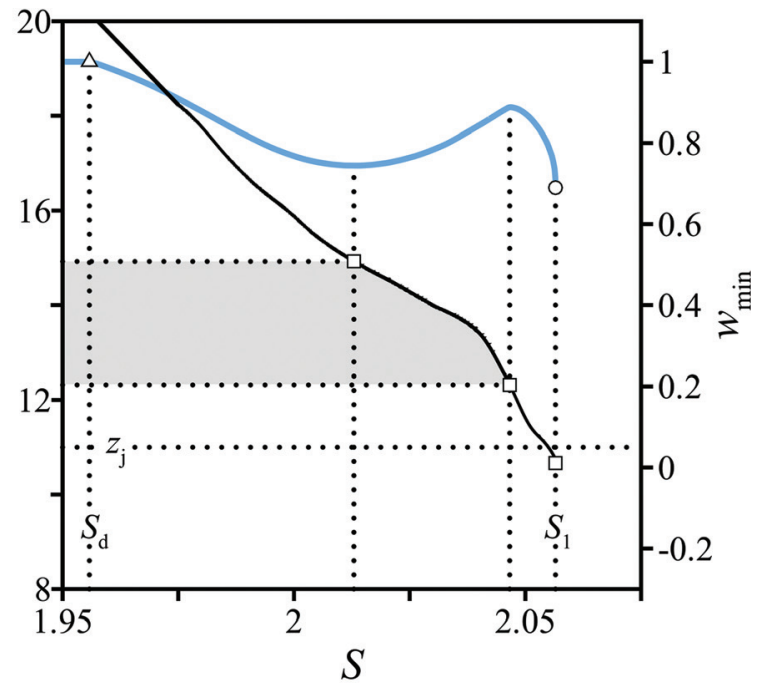

(b)

FIG. 11. (Color) Evolution of $z_{\min }$ as a function of the swirl number, $R e=500$. This specific position marks the center of the low-velocity region ultimately degenerating into the primary recirculating bubble. (a) Uncontrolled flow: the bifurcation curve of the columnar solution is reported from Fig. 3 as the thick black line and the position at which vortex breakdown occurs is indicated by the square symbol. (b) Controlled flow: the wall jet is located at $z_{\mathrm{j}}=11$ and the bifurcation curve of the columnar solution is reported from Fig. 9(b) as the thick light blue line. The grey shaded area indicates the range of positions $z_{\text {min }}$ over which the flow undergoes an acceleration, whose lower and upper bounds are marked by the leftmost square symbols. The position at which vortex breakdown occurs is indicated by the rightmost square symbol- $\eta=3 \%$.

low-velocity region and to prevent its further development, which results in the observed acceleration. This confinement ceases in the downstream vicinity of the injection section. In return, the flow decelerates again and breakdown ultimately occurs immediately after the low-velocity region has crossed the injection section (rightmost square symbol in Fig. 11(b)). Nevertheless, such a scenario is not generic, as can be inferred from the variety of bifurcation curves documented in Fig. 9. In practice, vortex downstream is altered in a very subtle way that depends on the strength of the deceleration achieved by the time the low-velocity region enters the region of influence of the jet. If the deceleration is already too severe (i.e., if the value of $w_{\min }$ is small positive), the confinement effect may be too weak for the flow to accelerate. In this case, the jet only slows down the upstream propagation of the low-velocity region until vortex breakdown finally occurs, which may explain the occurrence of smooth transitions.

\section{Alleviating hysteresis}

An alternative target of the control can be to minimize the range of swirl sustaining hysteresis, without paying attention to the individual domains of existence of the columnar and breakdown states. To this end, we simply extract the value of the critical swirls $S_{1}$ and $S_{2}$ from the various circle symbols reported in the bifurcation diagrams and define the optimal jet position as

$$
\Delta S_{\mathrm{opt}}=\min _{z_{\mathrm{j}}}\left\{S_{1}-S_{2}\right\} \equiv \Delta S\left(z_{\mathrm{j}, \mathrm{opt}}\right) .
$$

It can be concluded from Fig. 9 that there exist multiple optimal positions since the width of the hysteresis loop is precisely zero in the range $8.3 \leq z_{\mathrm{j}} \leq 9$ yielding a smooth transition. This is further illustrated in Fig. 12, where we report the upper and lower bounds of the hysteresis loop computed as a function of the jet position. The circle symbols stand for the computational data, whereas the light grey shaded area obtained by spline interpolation of the connecting lines represents the footprint of the domain in which the flow sustains hysteresis.

\section{Optimizing the topology of the columnar solution}

An important result issuing from the exhaustive nonlinear computations is that vortex breakdown occurs whatever

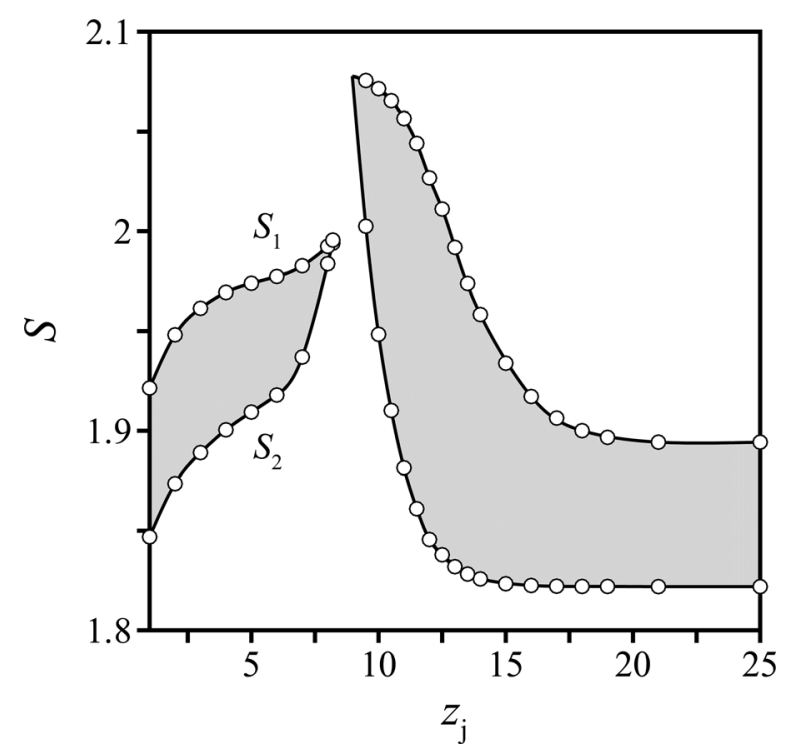

FIG. 12. Critical swirls $S_{1}$ and $S_{2}$ computed as a function of the jet position. The position for which $\Delta S=S_{1}-S_{2}$ is minimum defines the optimal jet position for a control aiming at alleviating hysteresis. For both plots, the circle symbols denote the actual computational data points, whereas the connecting lines have been obtained by spline interpolation. The light grey shaded area in between is the footprint of the domain in which the flow sustains hysteresis- $\eta=3 \%, R e=500$. 


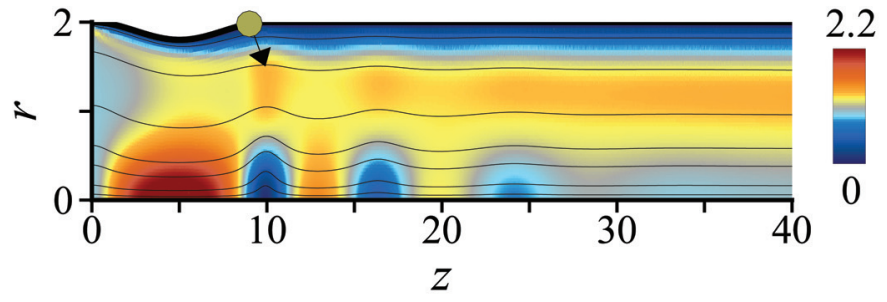

(a)

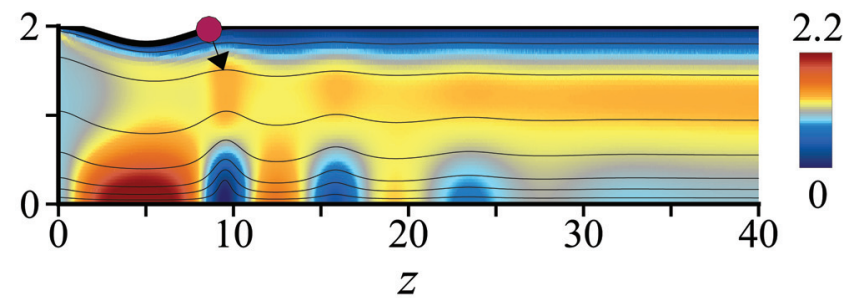

(b)

FIG. 13. (Color) Spatial distribution of axial velocity and characteristic streamline patterns for two representative solutions- $S=2.06, R e=500$. The position of the jet is indicated by the coloured symbols at the pipe wall. (a) $z_{\mathrm{j}}=9$ : the flow is on the verge of vortex breakdown. (b) $z_{\mathrm{j}}=8.6$ : breakdown has already occurred.

jet position is used. The relevance of a control concerned with the only stability of the columnar solution is therefore questionable in the perspective of applications where the jet is to be operated at a prescribed swirl number. Depending on the parameter settings, the columnar solution may indeed be stable but still be on the verge of breakdown. For instance, the topology of the columnar solution obtained for $S=2.06$ and $z_{\mathrm{j}}=9$ involves a series of subsequent low-velocity regions and differs very little from that of the breakdown solution prevailing at the same swirl for $z_{\mathrm{j}}=8.6$ (Fig. 13).

Consequently, the control target can also be to maximize the magnitude of axial velocity of the columnar solution: the major difference with respect to the previous approaches lies in the fact that the swirl is now viewed as a fixed parameter, our aim being to determine the new optimal position defined by

$$
\max _{z_{\mathrm{j}}}\left\{w_{\min } \mid S=\mathrm{cst}\right\} \equiv w_{\min }\left(z_{\mathrm{j}, \mathrm{opt}}, S\right) .
$$

In order to discuss the effect of the control, it is relevant to define first the swirl $S_{\mathrm{d} \text {,opt }}$ according to

$$
S_{\mathrm{d}, \mathrm{opt}}=\max _{z_{\mathrm{j}}}\left\{S_{\mathrm{d}}\right\} \equiv S_{\mathrm{d}}\left(z_{\mathrm{j}, \mathrm{opt}}\right),
$$

which yields here $S_{\mathrm{d} \text {,opt }}=1.973$, as can be extracted from the values of $S_{\mathrm{d}}$ reported in Fig. 9 as the various triangle symbols. By construction, there exists at least one jet location allowing to restore a fully columnar solution provided the operating swirl lies in the range $S<S_{\mathrm{d} \text {,opt }}$. As an example, we present in Fig. 14(a) the value of $w_{\min }$ computed as a function of the jet position for $S=1.94<S_{\mathrm{d} \text {,opt }}$, only the positive values being reported as the circle symbols. We find that the jet restores a stable columnar solution if set in a wide range $2 \leq z_{\mathrm{j}} \leq 14$. This is not surprising since the swirl is only slightly above the uncontrolled, critical value $S_{1 \text {,ini }}=1.893$. More interestingly, the control authority is excellent since the jet restores a fully columnar solution if set in the range $8 \leq z_{\mathrm{j} \text {,opt }} \leq 14$. However, the low value $w_{\text {min }}=0.18$ obtained for $z_{\mathrm{j}}=2$ warns that setting the jet to an arbitrary position may still yield a solution on the verge of breakdown. A second set of results obtained for $S=1.99>S_{\text {d,opt }}$ is presented in Fig. 14(b). Although no fully columnar solution can be obtained for this operating swirl, as could have been inferred from the above discussion, we find that the jet may still restore an almost fully columnar solution $\left(w_{\min }=0.95\right)$ if set at the optimal position $z_{\mathrm{j} \text {,opt }}=13$ indicated by the vertical dotted line. Nevertheless, the control authority has dropped dramatically: considering the range identified as being optimal for $S=1.94$ and reported as the light grey shaded area, we find now that $w_{\text {min }}=0.41$ for

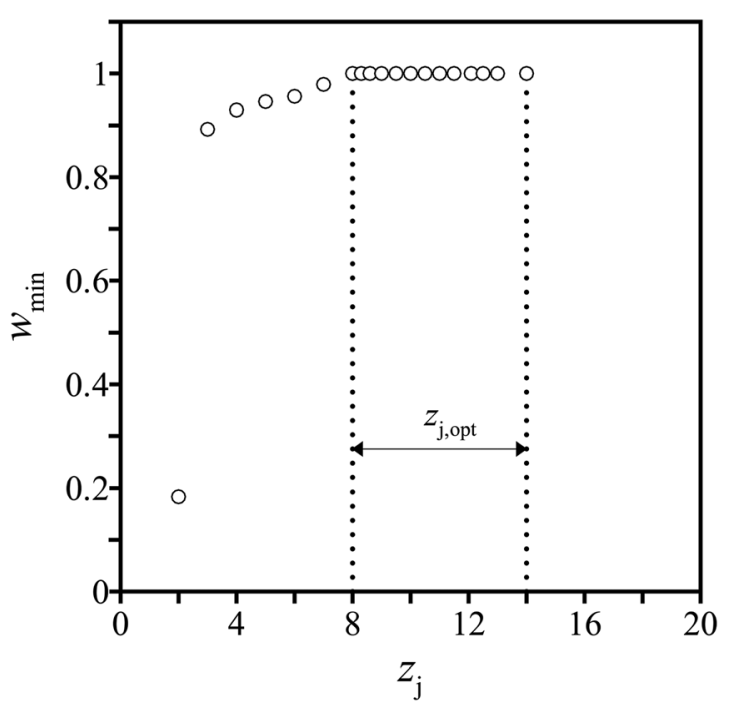

(a)

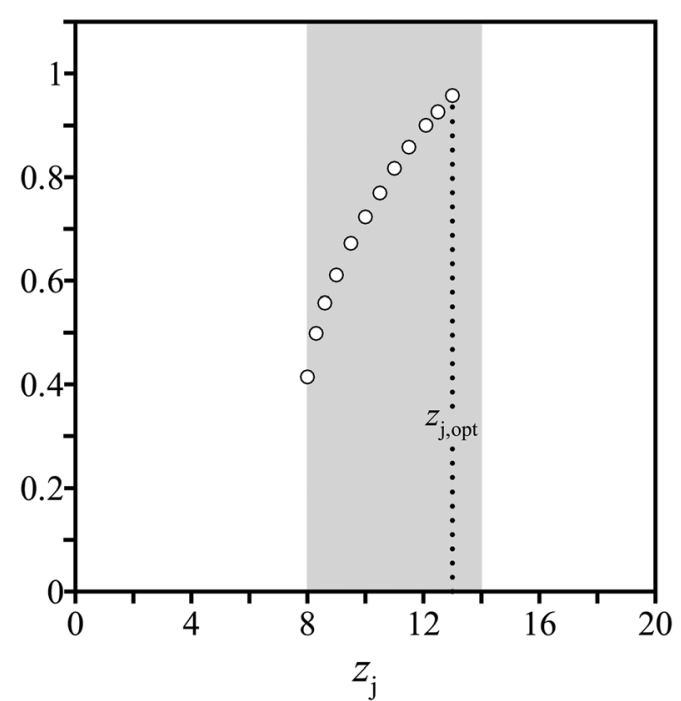

(b)

FIG. 14. Magnitude of the minimum velocity $w_{\min }$ computed as a function of the jet position- $\eta=3 \%, R e=500$. The position for which $w_{\text {min }}$ is maximum defines the optimal jet position for a control aiming at optimizing the topology of the columnar solution at a prescribed swirl. (a) $S=1.94$. (b) $S=1.99$. The grey shaded area is the footprint of the domain identified as being optimal in (a). 


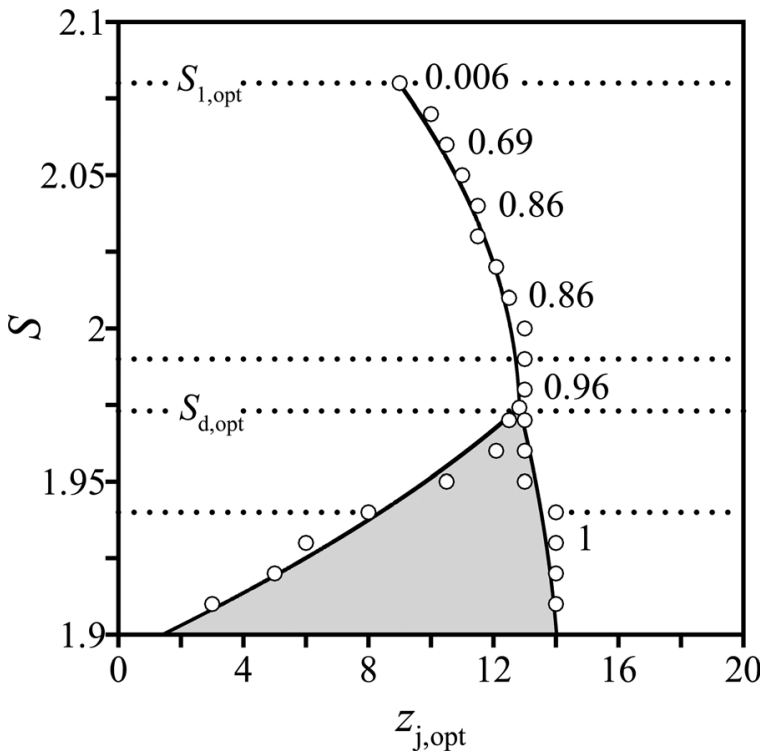

FIG. 15. (a) Optimal jet position $z_{\mathrm{j}, \mathrm{opt}}$ for a control meant to optimize the topology of the columnar solution, computed as a function of the operating swirl $-\eta=3 \% R e=500$. The circle symbols stand for the computational data points, whereas the solid lines have been obtained by polynomial interpolation. The grey shaded area corresponds to the range of positions within which the control restores a fully columnar solution $\left(w_{\min }=1\right)$. The unlabeled horizontal dotted lines mark the values $S=1.94$ and $S=1.99$ for which detailed results are presented in Fig. 14.

$z_{\mathrm{j}}=8$ and $w_{\min }<0$ for $z_{\mathrm{j}}=14$, a position for which no columnar solution can be recovered.

We have repeated this analysis by varying the operating swirl, the obtained optimal jet position being synthesized in Fig. 15. The computational data points are shown as the circle symbols along with the achieved value of $w_{\min }$ reported in the labels. The scattering of the data is due to the finite resolution $\Delta z_{\mathrm{j}}$ between two jet positions, so that we also provide qualitative curves obtained by polynomial interpolation. An optimal jet position can be found for which the level of axial velocity remains reasonably high provided $S \leq 2.05$, a value above which $w_{\text {min }}$ drops to very small positive values characterizing the initial stage of vortex breakdown. In any case, the operating swirl number cannot be increased above $S_{1, \mathrm{opt}}=2.08$ since the latter value precisely defines the swirl over which no columnar solution exists, whatever the jet position. If the jet is to be operated in this range, the only option is then to increase the jet flow rate, a point that will be addressed in Sec. V D.

\section{B. Limit of small control velocities and asymptotic expansion}

The results presented in Sec. V A have been obtained by undertaking exhaustive nonlinear calculations. In case the jet position is not motivated beforehand from any physical consideration, such an approach can be heuristic and particularly time-consuming, for instance, the derivation of the complete bifurcation diagrams has required the computation of about 3000 steady solutions.

As a mean to reduce the computational cost, we aim now at obtaining estimations of the exact results by use of a weakly nonlinear, asymptotic expansion. We consider first the uncontrolled flow and recall that the subcritical vortex breakdown identified in Sec. IV A coincides with the destabilization of the breakdown global mode. Assuming that the swirl varies in the vicinity of the critical value $S_{1 \text {,ini }}$, it is thus possible to asymptotically expand the solution around the marginally stable columnar state. Such an analysis aims at deriving an equation for the amplitude of the breakdown mode, denoted $A$. Two key points are worth being mentioned here:

- First, one must define a non-trivial dominant balance meant to set all effects at the same order. In the present case, we assume that a departure in the swirl at order $\epsilon^{2}$ forces disturbances of order $\epsilon$ to grow over a time scale of order $1 / \epsilon$.

- Second, the analysis requires to compute the adjoint of the bifurcating mode, hereafter denoted $\hat{\boldsymbol{q}}^{\dagger}$. The latter is used at order $\epsilon^{2}$ to enforce a compatibility condition whose role is to guarantee the existence of a solution to the expansion. ${ }^{42}$

We show in Appendix B that the amplitude $a=\epsilon A$ is governed by an amplitude equation

$$
\frac{\mathrm{d} a}{\mathrm{~d} t}=\alpha_{1} a^{2}+\beta_{1}\left(S_{1, \text { ini }}-S\right),
$$

characteristic of a saddle-node bifurcation, the variation of the swirl acting here as an offset whose magnitude is proportional to the departure from criticality. This contrasts with the theory developed by Rusak and co-workers, which rather leads to imperfect transcritical bifurcations, ${ }^{23-29}$ a point further discussed in Appendix B.

We also show in the Appendix that the real coefficients $\alpha_{1}$ and $\beta_{1}$ are related, respectively, to the self-transport of the breakdown mode and to the modification of the columnar solution as the swirl departs from criticality. Finally, we provide analytical expressions for these coefficients under the form of scalar products between the adjoint global mode and forcing terms of suitable amplitudes, derived from the marginally stable columnar state and breakdown mode. We obtain numerically

$$
\alpha_{1}=9.18 \times 10^{-3}, \quad \beta_{1}=-1.62 \times 10^{-1} .
$$

The branches of steady solutions prevailing in the vicinity of the critical swirl are obtained from Eq. (27) as

$$
a= \pm \sqrt{-\frac{\beta_{1}\left(S_{1, \mathrm{ini}}-S\right)}{\alpha_{1}}} .
$$

Since $\alpha_{1} \beta_{1}<0$, we retrieve that the bifurcation is subcritical,

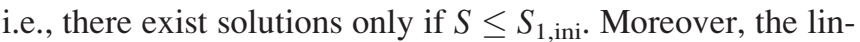
ear growth rate of the breakdown mode can be recovered by considering the stability of Eq. (29) to small-amplitude disturbances, which yields

$$
\sigma= \pm 2 \sqrt{-\alpha_{1} \beta_{1}\left(S_{1, \text { ini }}-S\right)}
$$

Figure 16 compares the growth rates predicted by these asymptotic relations, shown as the solid lines, to the direct stability calculations already presented in Fig. 5 and reported as 
the circle symbols. The agreement between both sets of results is excellent close to threshold. The difference is not even measurable for $S \geq 1.89$, hence validating the adjoint computations and the retained dominant balance. Small discrepancies exist for lower swirls, i.e., larger departure from criticality, where higher-order nonlinearities set in.

We return now to the controlled flow and assume that the growth of the flow disturbances is additionally forced at order $\epsilon$ by a control velocity of order $\epsilon^{2}$. We show in Appendix $B$ that in return, the amplitude Equation (27) is modified into

$$
\frac{\mathrm{d} a}{\mathrm{~d} t}=\alpha_{1} a^{2}+\beta_{1}\left(S_{1, \text { ini }}-S\right)+\gamma_{1} .
$$

In Eq. (31), $\gamma_{1}$ is the receptivity to the jet velocity, i.e., the real coefficient defined by

$$
\begin{aligned}
\gamma_{1}= & \int_{\partial \Sigma_{\mathrm{w}}}\left(\hat{p}^{\dagger}+\frac{1}{\operatorname{Re}}\left(\left(\nabla \hat{\boldsymbol{u}}^{\dagger}+\nabla \hat{\boldsymbol{u}}^{\dagger^{T}}\right) \cdot \boldsymbol{n}\right) \cdot \boldsymbol{n}\right) u_{\mathrm{c}}^{\perp} r \mathrm{~d} l / \\
& \int_{\Sigma} \hat{\boldsymbol{q}}^{\dagger} \cdot(\mathcal{B} \hat{\boldsymbol{q}}) r \mathrm{~d} r \mathrm{~d} z
\end{aligned}
$$

all quantities pertaining to the uncontrolled flow and being evaluated at criticality, i.e., at $S=S_{1, \text { ini. }}$ One sees from Eq. (32) that the $\gamma_{1}$ term stems from two distinct origins related to mass and viscous effects. The product of the wall-normal component of the velocity with the adjoint pressure taken at the wall accounts for the effect of the mass flux, whereas the contribution weighted by the inverse of the Reynolds number is due to the modification of the viscous force at the wall when the control velocity is applied. It has been argued in previous studies, ${ }^{43}$ and indeed checked in the present case, that the viscous term can be neglected compared to the effect of the mass flux for sufficiently large Reynolds numbers. This implies that the flow is receptive only to the wall-normal component of the velocity, hence motivating the retained approach.

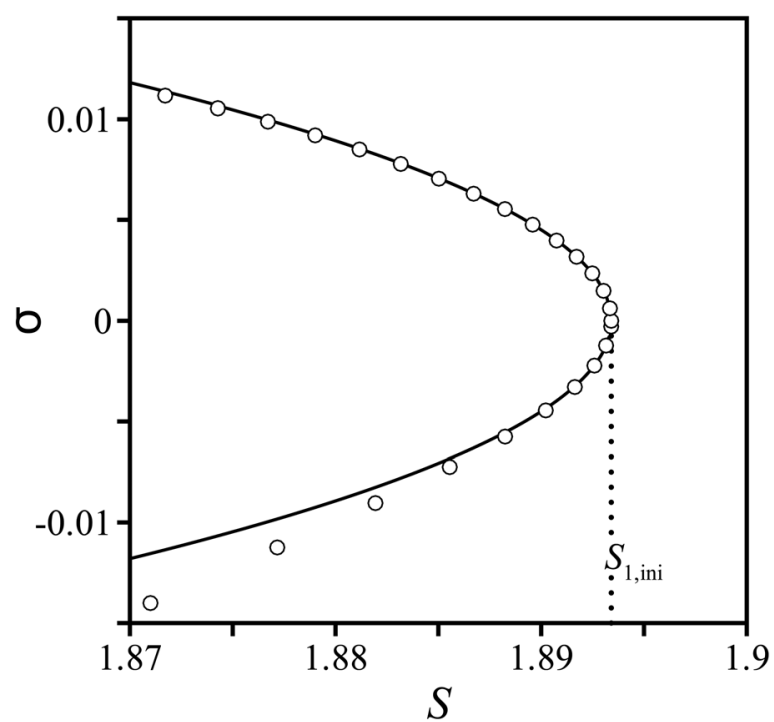

FIG. 16. Linear growth rate of the breakdown mode developing in the uncontrolled flow: comparison of the asymptotic values computed from Eq. (30) (solid lines) with the exact values obtained from the direct stability analysis and reported from Fig. 5 (circle symbols) $-R e=500$.
Note that the limit of small jet velocities is convenient to unravel the effect of the jet, which is seen to modify the bifurcation threshold by adding in a second offset term whose magnitude is independent of the swirl. The columnar solution (i.e., the solution at order $\epsilon^{0}$ ) and the breakdown mode (i.e., the solution at order $\epsilon$ ) are not affected directly since the effect of the externally applied control velocity is encompassed through the compatibility condition applied at order $\epsilon^{2}$. Nevertheless, the control directly adds on to the second-order resonant terms since the breakdown mode is steady and axisymmetric. On account of this resonance, the system amplifies the forcing by a factor $1 / \epsilon$, thus forcing the linear growth of the breakdown mode and its nonlinear saturation amplitude at order $\epsilon$.

\section{Comparison between asymptotic and nonlinear results}

We compare now the results issuing from the asymptotic approach presented in Sec. V B and that issuing from the exact nonlinear simulations carried out in Sec. V A. Note that we do not consider the last approach aiming at optimizing the topology of the columnar solution. Indeed, the criterion needed to assess the control effectiveness is very selective as it requires to determine the value of $w_{\min }$. In practice, one can compute the successive solutions arising at each order in $\epsilon$ and reconstruct an estimation of the nonlinear steady state from which the value of the axial velocity $w_{\text {min }}$ can be extracted. Nevertheless, the present expansions are truncated at a somewhat low order, hence yielding limited accuracy. From a more prospective point of view, quantitative results can be obtained using higher-order expansions, which, however, results in intricate computations lying out of the scope of the present study.

\section{Restoring the existence of the columnar solution}

In this section, we apply the asymptotic technique to the first control approach meant to restore the existence of the columnar solution. We recall that in this case, the effect of the control is quantified in terms of the critical swirl at which breakdown sets in. The branches of steady solutions issuing from Eq. (31) now read

$$
a= \pm \sqrt{-\frac{\beta_{1}\left(S_{1, \text { ini }}-S\right)+\gamma_{1}}{\alpha_{1}}}
$$

and the critical swirl $S_{1 \epsilon}$ is obtained by setting $a=0$ in Eq. (33), which yields

$$
S_{1 \epsilon}=S_{1, \text { ini }}+\frac{\gamma_{1}}{\beta_{1}}
$$

The main advantage of the asymptotic approach therefore lies in the fact that the effectiveness of the control in delaying vortex breakdown can be estimated from Eq. (34) through the computation of a limited number of scalar products, the knowledge of the controlled solution itself being no more required.

The adjoint pressure can be viewed from Eqs. (32)-(34) as a measure of the gradient of the critical swirl with respect to the jet velocity. In the present case, it is found to be 
negative for all axial positions along the wall (upper part of Fig. 17). Since $\beta_{1} \leq 0$, blowing fluid into the pipe $\left(u_{\mathrm{c}}^{\perp} \geq 0\right)$ guarantees that the stability of the columnar solution is restored beyond the threshold of global instability $\left(S_{1 \epsilon}>S_{1, \text { ini }}\right)$. Moreover, it can be inferred that $S_{1 \epsilon}$ reaches a maximum provided the jet is located at the position where the magnitude of adjoint pressure is the largest, namely, $z_{\mathrm{j} \epsilon \text {,opt }} \sim 12$ in the present case. This point is further illustrated in Fig. 18(a), where we have superimposed the asymptotic critical swirl $S_{1 \epsilon}$ for comparison with the exact results discussed in Sec. V A: we retrieve that the jet has a limited effect if located too close from the inlet section or too far downstream. The difference between both sets of results remains limited, the only noticeable effect being the shift in the optimal position, from $z_{\mathrm{j} \text {,opt }} \sim 9$ to $z_{\mathrm{j} \epsilon \text {,opt }} \sim 12$. This not too surprising since the optimal value $z_{\mathrm{j}, \mathrm{opt}}$ is precisely located in the range where the transition has been said to be smooth, and not subcritical.

\section{Alleviating hysteresis}

We consider now the second control approach meant to suppress hysteresis, for which the amplitude equation introduced above cannot be used straightforwardly. Indeed, the critical swirl $S_{2 \text {,ini }}$ corresponds to the threshold of instability of the breakdown solution. As a result, the asymptotic expansion originally undertaken to approximate the behaviour of the columnar solution in the vicinity of the critical swirl $S_{1, \text { ini }}$, herein referred to as the backward expansion, cannot be expected to bear any relevance. Consequently, we carry out a second expansion of the solution, the forward expansion, based on the marginally stable breakdown solution, whose aim is to derive a new equation for the amplitude of the breakdown mode in the vicinity of the critical swirl $S_{2 \text {,ini }}$. Although both expansions are almost identical, we insist that the breakdown mode develops from the columnar solution in the backward expansion and from the breakdown solution in the forward expansion, which constitutes a major difference from a physical point of view.

We show in Appendix B that this yields a new amplitude equation

$$
\frac{\mathrm{d} a}{\mathrm{~d} t}=\alpha_{2} a^{2}+\beta_{2}\left(S_{2, \text { ini }}-S\right)+\gamma_{2},
$$

with

$$
\alpha_{2}=-7.26 \times 10^{-3}, \quad \beta_{2}=-2.48 \times 10^{-1} .
$$

Note that the value of the $\gamma$ parameter differs in the backward and forward expansions since it depends not only on the control velocity but also from the adjoint pressure distribution, the latter being now evaluated at the critical swirl $S_{2 \text {,ini. Since }}$ $\alpha_{2} \beta_{2}>0$, we retrieve that the bifurcation is supercritical when no control is applied $\left(\gamma_{2}=0\right)$, i.e., there exist solutions

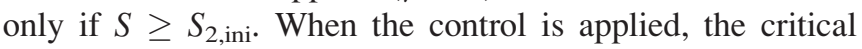
swirl at the forward turning point can now be obtained as

$$
S_{2 \epsilon}=S_{2, \text { ini }}+\frac{\gamma_{2}}{\beta_{2}}
$$

and the hysteresis range reads

$$
\Delta S_{\epsilon}=S_{1 \epsilon}-S_{2 \epsilon}=\Delta S_{\mathrm{ini}}+\frac{\gamma_{1}}{\beta_{1}}-\frac{\gamma_{2}}{\beta_{2}}
$$

This asymptotic range is shown in Fig. 18(b) to reproduce surprisingly well that issuing from the nonlinear steady-state computations. Moreover, there exist two positions of interest, namely, $z_{\mathrm{j}}=9$ and $z_{\mathrm{j}}=9.5$ for which the critical swirls coalesce, i.e., $\Delta S_{\epsilon}=0$. In terms of nonlinear bifurcation diagrams, this precisely defines the turnover from a subcritical to a smooth transition, such positions being in good agreement with the exact ones, namely, $z_{\mathrm{j}}=8.3$ and $z_{\mathrm{j}}=9.5$. Although agreement may be fortuitous, these results suggest that the simultaneous use of both asymptotic expansions allows to detect possible transitions to smooth vortex breakdown. Nevertheless, they remain unable to drive further quantitative predictions in case a turnover does occur, namely, relations (34)-(37) yield irrelevant values $\Delta S_{\epsilon}<0$ for jets located within the range $9 \leq z_{\mathrm{j}} \leq 9.5$, so that the associated data points are not reported in Fig. 18(b). This is
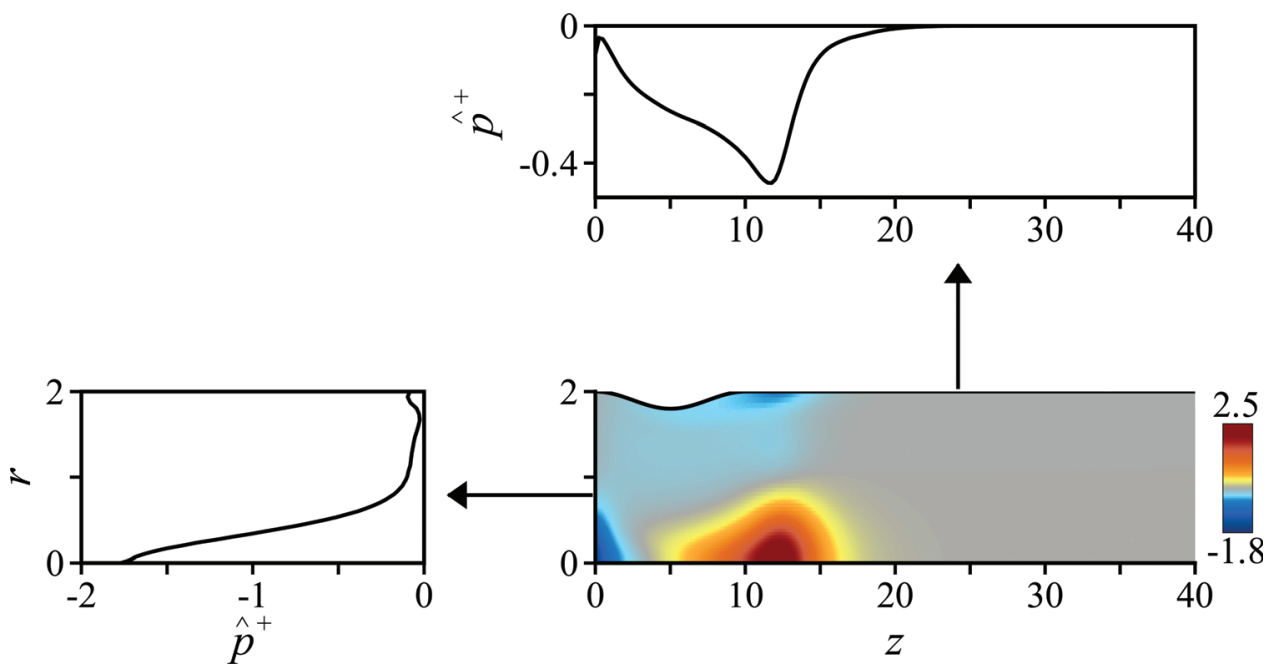

FIG. 17. (Color) Spatial distribution of adjoint pressure $\hat{p}^{\dagger}$ for the uncontrolled flow. The distribution is also plotted as a function of the streamwise position $z$ along the pipe wall (upper picture) and of the radial position $r$ in the inlet section (leftmost picture) $-S=1.893$, $\operatorname{Re}=500$. 


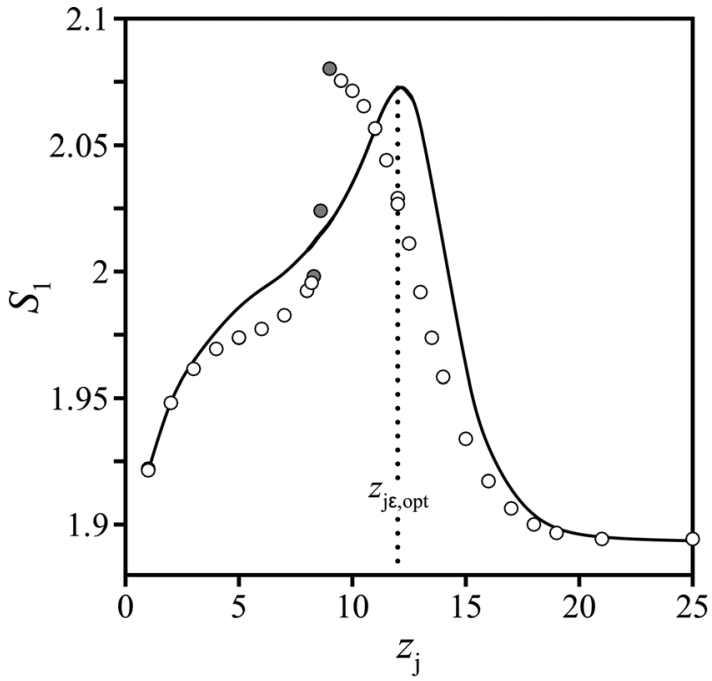

(a)

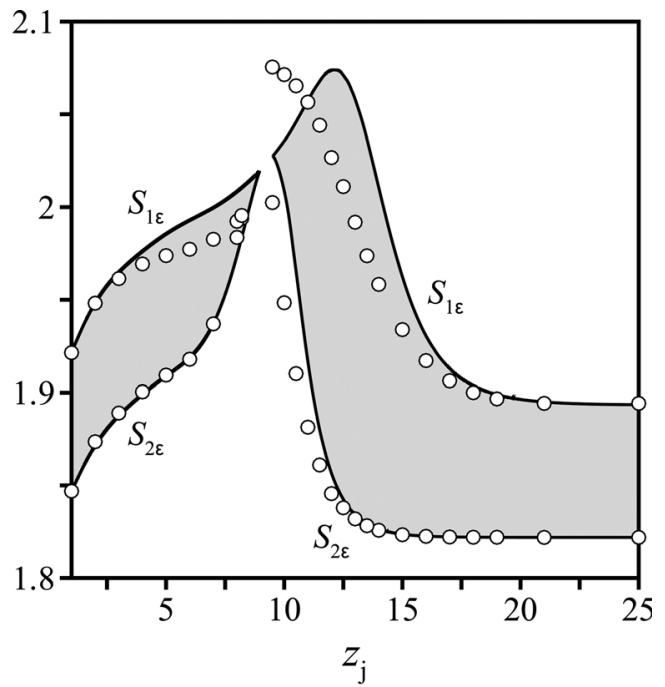

(b)

FIG. 18. (a) Asymptotic critical swirl $S_{1 \varepsilon}$ computed as a function of the jet position $z_{\mathrm{j}}$. The position for which $S_{1 \varepsilon}$ is maximum defines the optimal jet position for a control aiming at restoring the existence of the columnar solution. (b) Asymptotic critical swirls $S_{1 \varepsilon}$ and $S_{2 \varepsilon}$ computed as a function of the jet position. The position for which $\Delta S_{\varepsilon}=S_{1 \varepsilon}-S_{2 \varepsilon}$ is minimum defines the optimal jet position for a control aiming at alleviating hysteresis. For both plots, the circle symbols denote the data points issuing from the exact nonlinear steady state calculations already presented in Figs. 9,10 and $12-\eta=3 \%, R e=500$.

not surprising since the expansions intrinsically rely on the existence of bifurcation points, whereas the smooth transition arises without any loss of stability.

\section{Effect of the jet flow rate}

As an attempt to appraise the impact of nonlinearities on the controllability of vortex breakdown, we investigate now the effect of varying the flow rate of a jet located at $z_{\mathrm{j}}=12$, i.e., at the optimal position predicted by the adjoint-based asymptotic analysis. A complete bifurcation diagram has been derived for 5 values of $\eta$ in the range $\eta \leq 10 \%$, from which it turns out that vortex breakdown remains subcritical (not shown here for conciseness). We find that the critical swirl

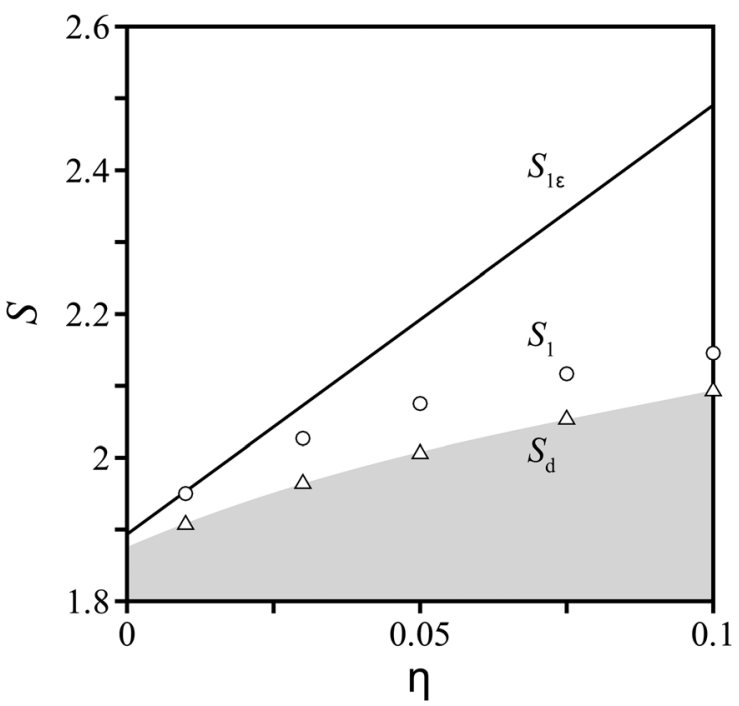

FIG. 19. Effect of the flow rate on the critical swirl $-R e=500$. The circle symbols denote the values issuing from nonlinear computations using a wall jet at the position $z_{\mathrm{j}}=12$, whereas the solid line indicates the asymptotic value computed from Eq. (34). The triangle symbols stand for the swirl $S_{\mathrm{d}}$ delimiting the domain of existence of a fully columnar solution. increases monotonically with the flow rate coefficient (circle symbols in Fig. 19), meaning that the range of operability of the jet can be improved by increasing the jet flow rate, as has been mentioned in Sec. V A. The asymptotic value $S_{1 \epsilon}$ shown as the solid line exhibits excellent agreement for low flow rates. For larger flow rates, the asymptotic approach increasingly overestimates the critical swirl, meaning that the control authority is limited by the nonlinearities. Still, the exact value is predicted reasonably well up to $\eta=5 \%$, the agreement being only in order of magnitude afterwards. This provides good evidence of the ability of the asymptotic approach to draw qualitative conclusions even at practically meaningful control settings. Note that the swirl $S_{\mathrm{d}}$ at which the solution ceases to be fully-columnar, also presented as the triangle symbols in Fig. 19, is found to increase similarly with the flow rate coefficient, such results being of practical interest for a control aiming at optimizing the topology of the columnar solution. The grey shaded area in Fig. 19 has been obtained by a spline interpolation of the connecting line and therefore represents the footprint of the domain in which the jet can be operated in the absence of any detrimental deceleration.

We propose now to define a quantitative measure of the net effectiveness by introducing the ratio

$$
\chi=\left(\frac{S_{1}}{S_{1, \text { ini }}}-1\right) \frac{1}{\eta} .
$$

For a wall jet allowing to delay vortex breakdown up to the critical value $S_{1}$, this $\chi$ parameter compares the jet flow rate (i.e., the cost of the control) to the flow rate that would have to be added uniformly at the pipe inlet in order to reach the same critical value, ${ }^{55}$ i.e., the control is effective only if it yields values $\chi>1$. The wall jet is found to be effective for all flow rates, namely, we obtain $\chi \sim 2.3$ for $\eta=3 \%$, meaning that one would need to increase uniformly the inlet flow rate by more than twice as much to reach the corresponding 
critical value. For larger values of $\eta$, the effectiveness is lower but still significant $(\chi=1.3$ for $\eta=10 \%)$. This can be explained by recalling that the effect of the control is then limited by the flow nonlinearities, whereas the increase in the inlet velocity acts through an affine rescaling of the swirl.

\section{CONTROL BY MODIFICATION OF THE INLET VELOCITY PROFILE}

As a mean to illustrate the importance of using physically motivated control strategies, we aim now at assessing the effect of an alternative control strategy relying on a manipulation of the axial velocity imposed at the pipe inlet. Such an approach seems particularly convenient since it is generally acknowledged that vortex breakdown is very sensitive to the choice of the upstream flow conditions. ${ }^{50,51}$

The control is designed from receptivity arguments only. Following the reasoning already held in Sec. V B, vortex breakdown is thus expected to occur close to its asymptotic value defined from Eq. (34), at least for small control velocities. Owing to the change in the control method, the $\gamma_{1}$ coefficient now reads

$$
\begin{aligned}
\gamma_{1}= & \int_{\partial \Sigma_{\mathrm{in}}}\left(\hat{p}^{\dagger}+\frac{1}{\operatorname{Re}}\left(\left(\nabla \hat{\boldsymbol{u}}^{\dagger}+\nabla \hat{\boldsymbol{u}}^{\dagger}\right) \cdot \boldsymbol{n}\right) \cdot \boldsymbol{n}\right) u_{\mathrm{c}}^{\perp} r \mathrm{~d} z / \\
& \int_{\Sigma} \hat{\boldsymbol{q}}^{\dagger} \cdot(\mathcal{B} \hat{\boldsymbol{q}}) r \mathrm{~d} r \mathrm{~d} z .
\end{aligned}
$$

The adjoint pressure taken at the inlet can be seen from Fig. 17 to be negative for all radial positions, hence confirming that the stability of the columnar solution can be restored beyond the threshold of instability by blowing fluid into the pipe. The control velocity is defined as $u_{\mathrm{c}}^{\perp}=-\mathrm{k} \hat{p}^{\dagger}$, where $\mathrm{k}>0$ is a normalization coefficient chosen so as to prescribe the relative variation in the total flow rate injected into the pipe, which now reads

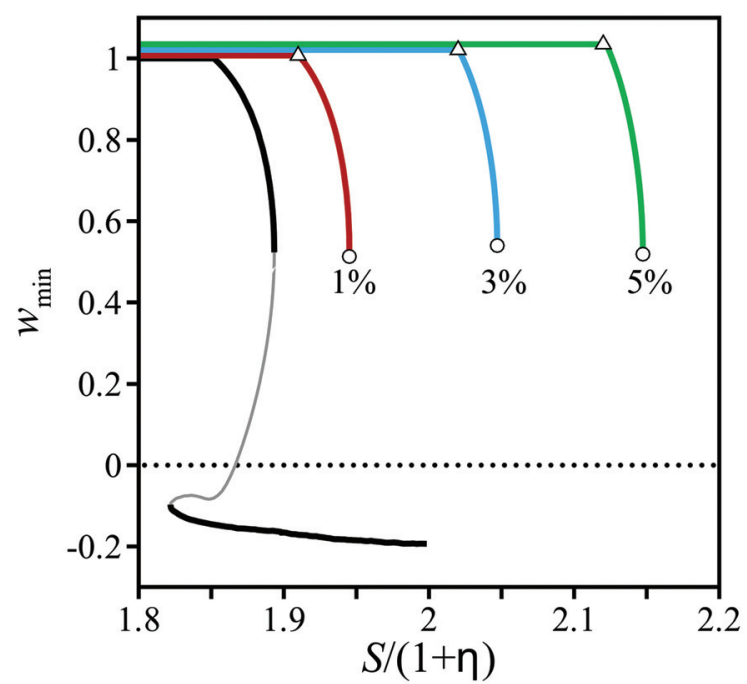

(a)

$$
\eta=\int_{\partial \Sigma_{\mathrm{in}}} u_{\mathrm{c}}^{\perp} r \mathrm{~d} r / \int_{\partial \Sigma_{\mathrm{in}}} w r \mathrm{~d} r .
$$

This distribution is simply meant for the control velocity to be large in the vortex core, i.e., in the region where the magnitude of adjoint pressure is maximum (Fig. 17) and small otherwise.

In the following, results are discussed in terms of a rescaled swirl $S /(1+\eta)$ built from the mean inlet velocity, which allows to assess the net effect of the control without taking into account the trivial increase of the mean flow rate. The obtained bifurcation diagrams are shown in Fig. 20, where we have restricted the presentation to the only columnar branch in order to ease the discussion. We find that vortex breakdown remains subcritical for moderate flow rates $\eta \leq 5 \%$ (open symbols), whereas it occurs through a smooth transition at larger flow rates $\eta \geq 7.5 \%$ (filled symbols). Moreover, the large nonlinear effects at work in the latter cases are seen to induce a significant distortion in the bifurcation curves.

The critical swirl increases monotonically with the flow rate coefficient (circle symbols in Fig. 21(a)), with a spectacular increase at large flow rate coefficients resulting from the onset of a smooth transition. An increase in the control flow rate therefore allows to improve the range of operability of the jet, as has been found for the wall jet. Moreover, the agreement with the asymptotic values shown as the solid line is excellent, the difference between both sets of results being barely visible as far as vortex breakdown remains subcritical. Comparing these results to those of the wall jet documented in Fig. 19, one sees that the value of $S_{1}$ obtained by a modification of the inlet velocity are significantly larger, hence suggesting that this new strategy is more effective at equal value of $\eta$, at least if the control is meant to restore the existence of the columnar solution. This is consistent with the fact that the

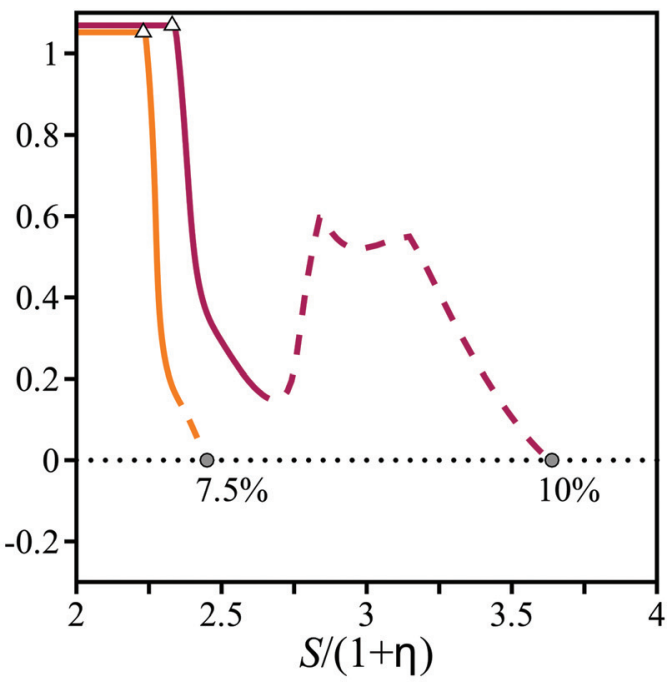

(b)

FIG. 20. (Color) Bifurcation diagrams of the columnar solution obtained by means of an adjoint-based manipulation of the inlet velocity profile- - Re $=500$. (a) Moderate flow rates $\eta<5 \%$. The black lines reproduce the bifurcation curve of the uncontrolled configuration. (b) Large flow rates $\eta>7.5 \%$. The circle symbols indicate the critical swirl $S_{1}$ at which vortex breakdown occurs (open symbols if subcritical, filled symbols if smooth), whereas the triangle symbols indicate the swirl $S_{\mathrm{d}}$ above which the fully columnar solution ceases to exist. The dashed lines in (b) correspond to swirl numbers for which the columnar solution is stable to axisymmetric disturbances, but unstable to helical disturbances, as discussed in Sec. VII. 


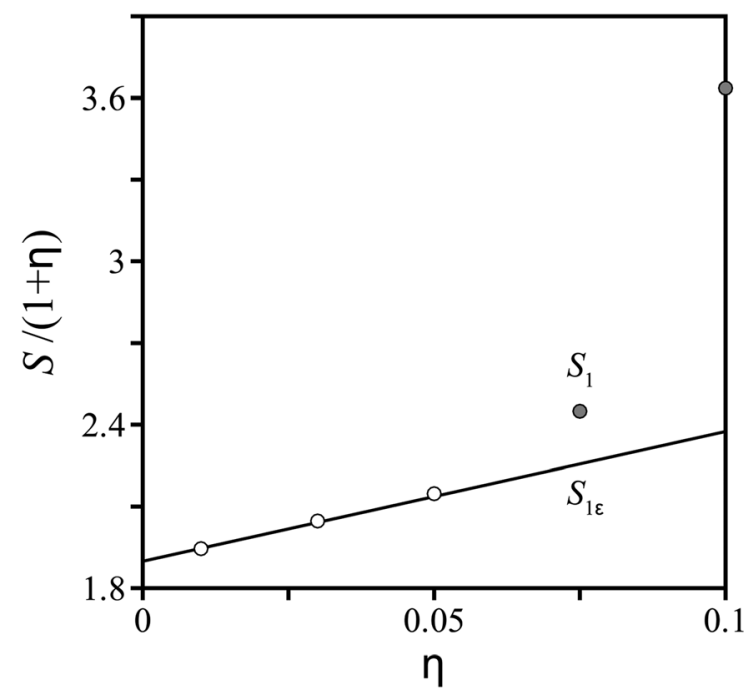

(a)

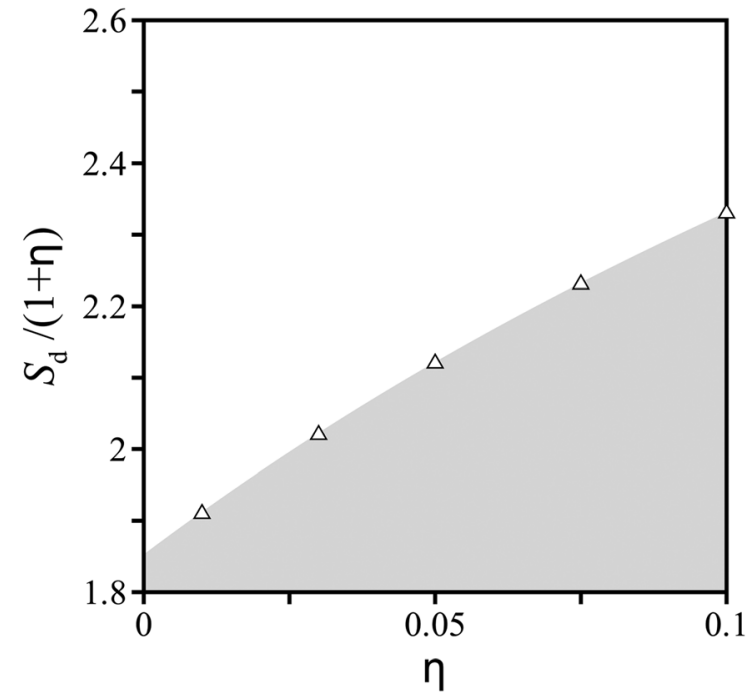

(b)

FIG. 21. Effect of a control relying on an adjoint-based manipulation of the inlet velocity profile- $R e=500$. (a) Critical swirl $S_{1}$ computed as a function of the control flow rate. Open symbols (respectively filled symbols) denote the value obtained from nonlinear simulations involving a subcritical transition to vortex breakdown (respectively a smooth transition), whereas the solid line corresponds to the asymptotic values computed from Eq. (34). (b) Evolution of the swirl $S_{\mathrm{d}}$ delimiting the domain of existence of a fully columnar solution.

level of adjoint pressure, i.e., the level of receptivity to the control velocity, is significantly larger at the inlet than at the pipe wall, as seen from Fig. 17. Similarly, we find that the value of the swirl $S_{\mathrm{d}}$ at which the solution ceases to be fullycolumnar (triangle symbols in Fig. 21(b)) is significantly larger than that obtained with the wall jet (Fig. 19), hence suggesting that the present approach may also be more effective if the control is meant to optimize the topology of the columnar solution. Finally, it is also more effective in terms of the $\chi$ coefficient: for instance, for $\eta=3 \%$, we find $\chi=3.8$, meaning that the uniform increase in the inlet flow rate needed to achieve the same critical swirl is almost four times as much.

\section{DISCUSSION: AXISYMMETRIC MODEL VS. NON-AXISYMMETRIC DYNAMICS}

In concluding this study, it may be worth recalling that all results presented so far have been obtained under the generally acknowledged assumption that vortex breakdown can be characterized in terms of an axisymmetric model. The present section briefly discusses the relevance of this approach by focusing on the large-time, non-axisymmetric dynamics, which can be straightforwardly interpreted from the existence of unstable global modes. Discussing the short-time dynamics also bears physical relevance, especially since vortex flows are well-known to sustain convective instability over large ranges of azimuthal wavenumbers, the latter being responsible for the ability to exhibit large transient amplifications of an initial condition. Nevertheless, this would require to carry out three-dimensional, optimal perturbation analyses, which cannot be handled by means of our numerical tools.

We have analyzed the stability of the various branches of solutions to helical disturbances of azimuthal wavenumbers $1 \leq|m| \leq 3$, the calculations being restricted without loss of generality to eigenvalues in the $\omega \geq 0$ half-plane. When solving the corresponding eigenvalue problem (15), this requires to replace the axisymmetric linearized evolution operator $\mathcal{A}$ by its general form in which all $\theta$ derivatives are replaced by products by im, and to adapt the boundary condition at the revolution axis from the mass and momentum conservation. ${ }^{34}$ Fig. 22 presents the growth rate of the leading eigenmodes monitored as a function of the swirl for the uncontrolled columnar and breakdown solutions, respectively. One sees from Fig. 22(a) that the columnar solution remains stable to any disturbances up to the critical value $S_{1, \text { ini }}$. In contrast, the breakdown solution is immediately unstable to a branch of $m=-1$ modes at the critical swirl $S_{2, \text { ini }}$, and we find an additional branch of unstable $m=-2$ modes for $S>1.885$.

These results suggest that vortex breakdown as observed from an experimental set-up may involve a steady axisymmetric pattern persisting over a finite time before being altered by the subsequent development of disturbances of various azimuthal wavenumbers. This singularizes out axisymmetric vortex breakdown as being a prerequisite to the existence of any other pattern, a conclusion reminiscent of that proposed by Ruith et al. ${ }^{10}$ in the case of an unconfined vortex. Interestingly enough, the wavenumber selection issuing from our analysis is consistent with that documented by these authors, which relies on the assumption that the selfsustained oscillations are due to the wake-like axial velocity profiles of the axisymmetric breakdown solution being absolutely unstable. The same remark holds for the frequency selection as we find typical frequencies of order $S t=\omega / 2 \pi \sim 0.2$ for the $m=-1$ mode and $S t \sim 0.4$ for the $m=-2$ mode (not shown here for conciseness).

The stability of the various controlled columnar states has been similarly analyzed for a restricted number of swirl numbers, including the critical value $S_{1}$. When control is achieved by means of a wall jet, all solutions have been 


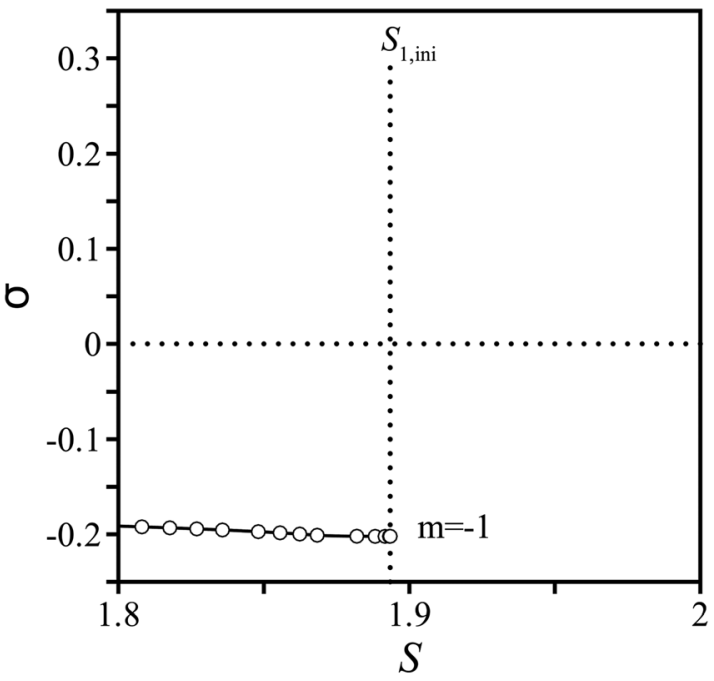

(a)

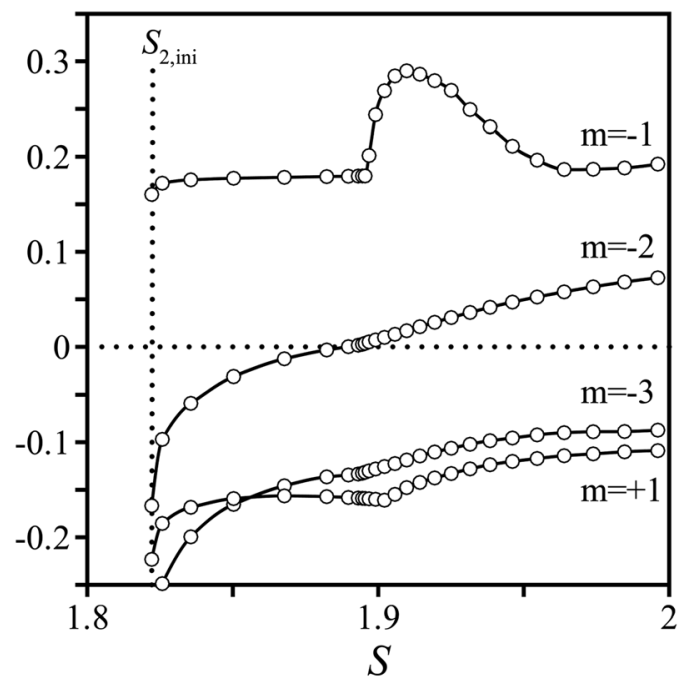

(b)

FIG. 22. Stability of the uncontrolled axisymmetric solutions to helical disturbances of azimuthal wavenumbers- $R e=500$. The growth rate of the leading eigenmode is reported for each wavenumber in the range $1 \leq|m| \leq 3$. (a) Columnar solution. (b) Breakdown solution.

found to be stable to helical disturbances up to the critical swirl $S_{1}$. When control is achieved through a modification of the inlet profile, only for the two largest flow rate coefficients $(\eta \geq 7.5 \%)$ does a helical instability set prior to axisymmetric vortex breakdown (the corresponding bifurcation curves being then shown as dashed lines in Fig. 20), hence illustrating the generality of the present conclusions.

\section{CONCLUSION}

We have investigated numerically and theoretically the bifurcation structure leading to axisymmetric vortex breakdown in a contracted pipe of infinite extension. A numerical continuation method has been developed to compute branches of steady axisymmetric solutions as a function of the swirl number. Various flow patterns characteristic of the columnar, decelerated, and breakdown states have been identified. We have shown that in the most general case, vortex breakdown can be interpreted as the consequence of a fold of the non-parallel columnar solution related to the subcritical destabilization of a global eigenmode, hence explaining the generally acknowledged hysteretic behaviour sustained by this class of flows. When the vortex core is strongly confined within the pipe, a smooth transition is, however, possible without any loss of stability.

We have also addressed the question of flow control by characterizing the effect of a localized jet positioned at the pipe wall. A parametric study has been carried out in terms of the jet position and flow rate. For each control setting, nonlinear numerical simulations have been performed and have provided evidence that an optimal jet position can be found, whose precise value depends on the control target. Three cases have been considered: in the first one, the control simply aims at maximizing the critical swirl at which vortex breakdown occurs, without paying attention to the flow topology; in the second one, it aims at suppressing hysteresis; in the third one, it aims at maximizing the axial velocity of the columnar solution for a prescribed operating swirl. All three approaches singularize out the vicinity of the contraction out- let as the pipe region where maximum effectiveness is achieved. The nonlinear results have also been shown to compare satisfactorily to those issuing from an asymptotic expansion of the marginally stable solutions. This technique stands as a promising tool for the design of future control laws, even at realistic parameter settings. Indeed, it requires the only computation of an adjoint global mode and allows to draw qualitative predictions with no need to compute the controlled solutions themselves. As an illustration, we have shown that the effect of the wall jet can be outdone by an adjoint-based modification of the axial velocity profile at the pipe inlet. In return, fully columnar solutions can be retrieved over a large range of swirl exceeding that above which the wall jet succeeds in restoring its only stability. Nevertheless, several issues remain to be addressed in the perspective of real flow applications; in particular, further efforts should be devoted to assess the ability of the control to alleviate the detrimental effects of vortex breakdown from fully three-dimensional numerical simulations.

\section{ACKNOWLEDGMENTS}

The authors warmly acknowledge the help of D. Guy and the computational facilities at LadHyX (CNRS-Ecole Polytechnique).

\section{APPENDIX A: SENSITIVITY RESULTS TO MESH SPACING}

In order to assess convergence in the numerical results, complete bifurcation diagrams have been computed for five meshes $M_{1}$ to $M_{5}$ and three configurations:

- the nominal configuration,

- the wall-jet configuration for $z_{\mathrm{j}}=8.6$ and $\eta=3 \%$,

- the wall-jet configuration for $z_{\mathrm{j}}=11$ and $\eta=3 \%$.

The meshes exhibit various spatial extents and vertex densities, as well as various settings in the sponge region, as detailed in Table I. 
TABLE I. Properties of the meshes as a function of the parameters $L_{\infty}$ and $l_{s}$, corresponding to the length of the computational pipe and to the size of the sponge region. $n_{t}$ is the number of triangles and $D o F$ is the number of degrees of freedom for three-dimensional state vectors. Meshes $M_{1}$ and $M_{2}$ have the same vertex densities and differ by the size of the pipe. $M_{1}$ and $M_{3}$ have the same spatial extent, but $M_{3}$ is built with larger vertex densities. $M_{1}$ and $M_{4}$ differ by the size of the sponge region. Finally, $M_{1}$ and $M_{5}$ are identical, but calculations on $M_{5}$ rely on a different damping function in the sponge region, defined by relation (9) along with $\varsigma=3$.

\begin{tabular}{ccccc}
\hline \hline & $L_{\infty}$ & $l_{s}$ & $n_{t}$ & $D o F$ \\
\hline$M_{1}$ & 50 & 35 & 84626 & 834404 \\
$M_{2}$ & 60 & 35 & 94244 & 929234 \\
$M_{3}$ & 50 & 35 & 114700 & 1133293 \\
$M_{4}$ & 50 & 45 & 94244 & 929234 \\
$M_{5}$ & 50 & 35 & 84626 & 834404 \\
\hline \hline
\end{tabular}

Results are presented in Fig. 23. For each configuration, the bifurcation curve documented in the article body, pertaining to mesh $M_{1}$, is reported as the solid line. The data issuing from the present sensitivity analysis, pertaining to meshes $M_{2}-M_{5}$, are superimposed as the circle symbols. In all three cases, the change in the discretization is seen to have a very limited effect. Confirmation comes from Table II, where we report numerical values of the critical swirl extracted from these diagrams, namely, we report $S_{\mathrm{b}}$ and $S_{\mathrm{f}}$ if the transition to vortex breakdown is subcritical and $S_{0}$ if the transition is smooth. A very good convergence is seen to be achieved since all values remain identical down to the second digit. In particular, it is worthwhile noting that the results are insensitive to the use of sponge regions.

\section{APPENDIX B: ASYMPTOTIC EXPANSION OF THE NEARLY CRITICAL STATES}

This appendix is devoted to the derivation of the amplitude equations used in Sec. V B. We define here a generic critical swirl $S_{*}$ that stands either for the primary value $S_{1, \text { ini }}$ (backward expansion) or the secondary value $S_{2, \text { ini }}$ (forward expansion). As mentioned in the article body, we define a dominant balance meant to bring the linear growth of disturbances induced by a departure from threshold at the same order as the nonlinear term resulting from the self-transport of the perturbation. Namely, we assume that the swirl departs from criticality at order $\epsilon^{2}$ and introduce the order unity quantity $\Delta$ such that

$$
S=S_{*}-\epsilon^{2} \Delta .
$$

We also introduce multiple time scales with a fast time scale $t$ and a slow time scale $T=\epsilon t$, so that the time-dependent term in the governing equations is transformed according to

$$
\frac{\partial}{\partial t} \rightarrow \frac{\partial}{\partial t}+\epsilon \frac{\partial}{\partial T} .
$$

\section{Uncontrolled case}

The solution is expanded into

$$
\boldsymbol{q}=\boldsymbol{q}(0)+\epsilon \boldsymbol{q}_{(1)}+\epsilon^{2} \boldsymbol{q}_{(2)}+\cdots .
$$
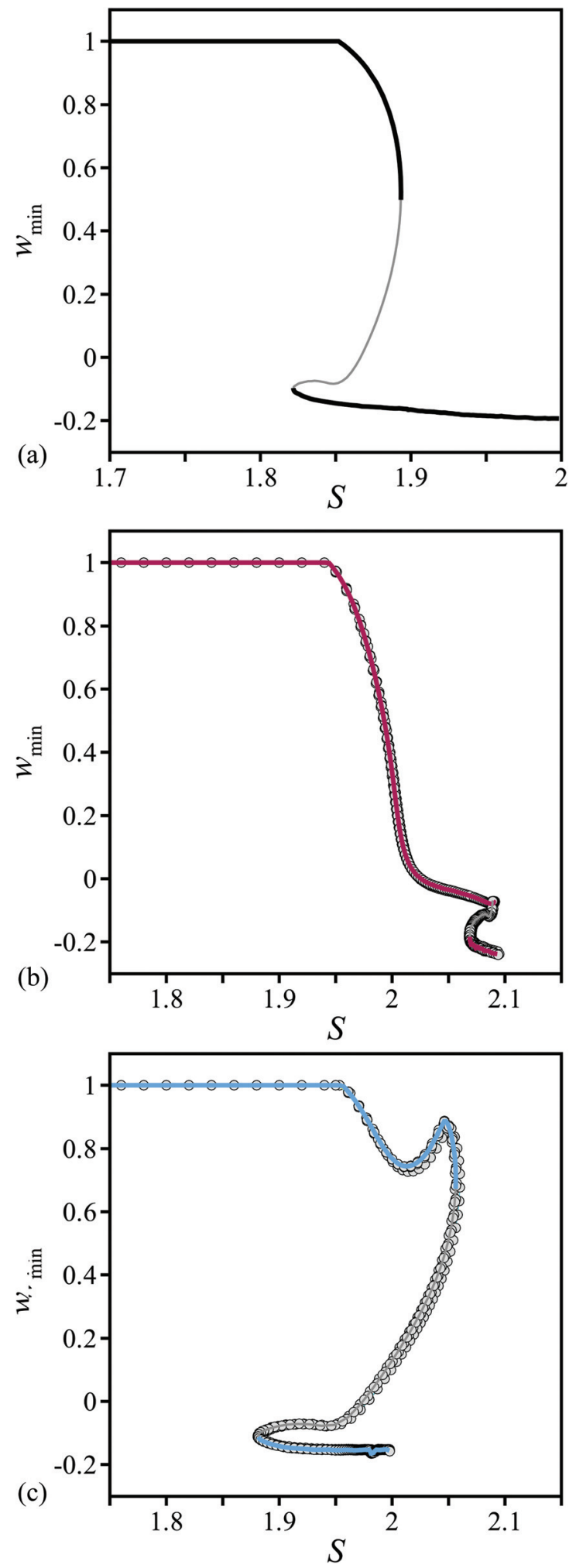

FIG. 23. (Color) Bifurcation diagrams obtained using the five meshes characterized in Table I. Results pertaining to mesh $M_{1}$ are replicated from Figs. 3 and 9 and correspond to the solid lines. Results pertaining to meshes $M_{2}-M_{5}$ are superimposed as the circle symbols $-R e=500$. (a) Nominal configuration. (b) Wall-jet configuration- $z_{\mathrm{j}}=8.6, \eta=3 \%$. (c) Wall-jet configuration $-z_{\mathrm{j}}=11, \eta=3 \%$.

Substitution of the previous expansions into Eq. (10) yields a series of equations at successive order of $\epsilon$. The equations at order $\epsilon^{0}$ are the steady, nonlinear Navier-Stokes equations (11) written at the critical swirl 
TABLE II. Sensitivity of the critical swirl to the different meshes characterized in Table $\mathrm{I}-\operatorname{Re}=500$.

\begin{tabular}{|c|c|c|c|c|c|}
\hline & \multicolumn{2}{|c|}{ Nominal } & \multirow{3}{*}{$\begin{array}{c}\text { Wall jet } \\
z_{\mathrm{j}}=8.6, \eta=3 \% \\
S_{0}\end{array}$} & \multirow{2}{*}{\multicolumn{2}{|c|}{$\begin{array}{c}\text { Wall jet } \\
z_{\mathrm{j}}=11, \eta=3 \%\end{array}$}} \\
\hline & \multirow[b]{2}{*}{$S_{\mathrm{b}}$} & \multirow[b]{2}{*}{$S_{\mathrm{f}}$} & & & \\
\hline & & & & $S_{\mathrm{b}}$ & $S_{\mathrm{f}}$ \\
\hline$M_{1}$ & 1.8934 & 1.8219 & 2.0257 & 2.0565 & 1.8813 \\
\hline$M_{2}$ & 1.8939 & 1.8222 & 2.0256 & 2.0570 & 1.8819 \\
\hline$M_{3}$ & 1.8956 & 1.8246 & 2.0243 & 2.0601 & 1.8848 \\
\hline$M_{4}$ & 1.8940 & 1.8223 & 2.0259 & 2.0571 & 1.8819 \\
\hline$M_{5}$ & 1.8934 & 1.8219 & 2.0257 & 2.0567 & 1.8816 \\
\hline
\end{tabular}

$$
\mathcal{M}\left(\boldsymbol{q}_{(0)}, S_{*}\right)=\mathbf{0},
$$

and define $\boldsymbol{q}_{(0)}$ as the marginally solution computed in Sec. IV A (columnar solution for the backward expansion, breakdown solution for the forward expansion). The equations at order $\epsilon$ are the linearized Navier-Stokes equations

$$
\mathcal{A}\left(\boldsymbol{q}_{(0)}, S_{*}\right) \boldsymbol{q}_{(1)}=\mathbf{0}
$$

and specify that $\boldsymbol{q}_{(1)}$ can be chosen as a superposition of eigenmodes destabilizing the columnar solution. Consequently, we set

$$
\boldsymbol{q}_{(1)}=A(T) \hat{\boldsymbol{q}}_{*},
$$

with the complex amplitude $A$ being, at this stage, an unknown function of the slow time $T$. The equations at order $\epsilon^{2}$ are the linearized Navier-Stokes equations applied to $\boldsymbol{q}_{(2)}$, forced by a steady, axisymmetric resonant forcing term $\boldsymbol{F}_{(2)}$ depending on the zeroth and first-order solutions

$$
\mathcal{A}\left(\boldsymbol{q}_{(0)}, S_{*}\right) \boldsymbol{q}_{(2)}=\boldsymbol{F}_{(2)},
$$

with

$$
\boldsymbol{F}_{(2)}=-\frac{\partial A}{\partial T}\left(\begin{array}{c}
0 \\
\hat{\boldsymbol{u}}
\end{array}\right)-A^{2}\left(\begin{array}{c}
0 \\
\nabla \hat{\boldsymbol{u}} \cdot \hat{\boldsymbol{u}}
\end{array}\right)-\Delta\left(\begin{array}{c}
0 \\
\frac{2 S_{*} v_{(0)}^{2}}{r} \boldsymbol{e}_{\boldsymbol{r}}
\end{array}\right) .
$$

The first term in Eq. (B8) is a slow variation term arising from the time-evolution of the unknown amplitude $A$. The second term is nonlinear and is due to the transport of the first-order solution by itself, whereas the last term is linear and arises from variation of the swirl acting here on the base flow solution.

At this stage, a compatibility condition must be applied for the expansion procedure to be solved. We use a standard Fredholm alternative, ${ }^{42}$ specifying that the resonant forcing term must be orthogonal to the kernel of the adjoint linearized Navier-Stokes operator, i.e.,

$$
\int_{\Sigma} \hat{\boldsymbol{q}}^{\dagger} \cdot \boldsymbol{F}_{(2)} r \mathrm{~d} r \mathrm{~d} z=0
$$

In Eq. (B9), $\hat{\boldsymbol{q}}^{\dagger}$ is the adjoint global mode solution of the adjoint eigenvalue problem

$$
\mathcal{A}^{\dagger}\left(\boldsymbol{q}_{(0)}, S_{*}\right) \hat{\boldsymbol{q}}^{\dagger}=\mathbf{0}
$$

where $\mathcal{A}^{\dagger}$ is the adjoint of operator $\mathcal{A}$ obtained by integrating by parts the disturbance equations. ${ }^{52}$

In practice, $\hat{\boldsymbol{q}}^{\dagger}$ is obtained using the Arnoldi method already used for the stability analyses. Substituting Eq. (B8) into Eq. (B9) yields the amplitude equation

$$
\frac{\mathrm{d} A}{\mathrm{~d} T}=\alpha A^{2}+\beta \Delta
$$

that can be recast in terms of the physical amplitude $a=\epsilon A$ according to

$$
\frac{\mathrm{d} a}{\mathrm{~d} t}=\alpha a^{2}+\beta\left(S_{*}-S\right) .
$$

In Eqs. (B11) and (B12), $\alpha$ and $\beta$ are the real coefficients defined by

$$
\begin{aligned}
& \alpha=-\frac{1}{\mathcal{S}} \int_{\Sigma} \hat{\boldsymbol{u}}^{\dagger} \cdot(\nabla \hat{\boldsymbol{u}} \cdot \hat{\boldsymbol{u}}) r \mathrm{~d} r \mathrm{~d} z, \\
& \beta=-\frac{1}{\mathcal{S}} \int_{\Sigma} \hat{\boldsymbol{u}}^{\dagger} \cdot \frac{2 S_{*} v_{(0)}^{2}}{r} \boldsymbol{e}_{r} r \mathrm{~d} r \mathrm{~d} z,
\end{aligned}
$$

where we note

$$
\mathcal{S}=\int_{\Sigma} \hat{\boldsymbol{q}}^{\dagger} \cdot(\mathcal{B} \hat{\boldsymbol{q}}) r \mathrm{~d} r \mathrm{~d} z
$$

We have mentioned in the article body that Eq. (B11) characterizes a saddle-node bifurcation, for which the variation of the swirl acts here as an offset whose magnitude is proportional to the departure from criticality. The latter differs from the transcritical bifurcation model proposed by Rusak and co-workers, for which the variation of the swirl acts by adding in a term proportional both to the eigenmode amplitude and to the departure from criticality. Using the present balance, such a term comes at order $\epsilon^{3}$ in the expansion, i.e., it can be neglected rigorously when applying the compatibility condition at order $\epsilon^{2}$, but can be retrieved by truncating the expansion at a higher order, which however falls out of the scope of the paper.

For a similar term to come at order $\epsilon^{2}$, a different balance is required, namely, the departure from criticality must be chosen of order $\epsilon$. In return, the equations at order $\epsilon$ are the linearized Navier-Stokes equations, forced by the term extracted from Eq. (B8) and characterizing the effect of varying the swirl on the base flow solution

$$
\mathcal{A}\left(\boldsymbol{q}_{(0)}, S_{*}\right) \boldsymbol{q}_{(1)}=\boldsymbol{F}_{(1)}=-\Delta\left(\begin{array}{c}
0 \\
\frac{2 S_{*} v_{(0)}^{2}}{r} \boldsymbol{e}_{\boldsymbol{r}}
\end{array}\right) .
$$

Applying the compatibility condition

$$
\int_{\Sigma} \hat{\boldsymbol{q}}^{\dagger} \cdot F_{(1)} r \mathrm{~d} r \mathrm{~d} z=0
$$

one is left with the trivial relation $\beta=0$. Nevertheless, the value of the $\beta$ coefficient depends on the adjoint global mode and on the base flow solution, but not on the balance itself, and we recall that the values computed for the present case are $\beta_{1}=-1.62 \times 10^{-1}$ (backward expansion) and 
$\beta_{2}=-2.48 \times 10^{-1}$ (forward expansion). This inconsistence indicates that such an expansion would admit no solution, in contrast with the theory of Rusak and co-workers. It can be explained by recalling that the asymptotic expansions documented by these authors rely on an inviscid, parallel columnar solution, whereas the present one is viscous and non-parallel in essence. Our expansion is thus to be viewed as a close-up on the perturbed transcritical solution predicted by these authors, for which a different scaling is required.

\section{Controlled case}

We assume now a control velocity of order $\epsilon^{2}$ and introduce the order unity quantity $U_{\mathrm{c}}^{\perp}$ such that

$$
u_{\mathrm{c}}^{\perp}=\epsilon^{2} U_{\mathrm{c}}^{\perp} .
$$

Owing to this balance, the control velocity directly adds on to the resonant terms at order $\epsilon^{2}$. In this case, relation (B9) is not valid anymore, and the scalar product between the adjoint global mode and the second-order forcing term reads

$$
\begin{aligned}
\int_{\Sigma} \hat{\boldsymbol{q}}^{\dagger} \cdot \boldsymbol{F}_{(2)} r \mathrm{~d} r \mathrm{~d} z & =\int_{\Sigma} \hat{\boldsymbol{q}}^{\dagger} \cdot \mathcal{A}_{*} \boldsymbol{q}_{(2)} r \mathrm{~d} r \mathrm{~d} z \\
& =\underbrace{\int_{\Sigma} \mathcal{A}_{*}^{\dagger} \hat{\boldsymbol{q}}^{\dagger} \cdot \boldsymbol{q}_{(2)} r \mathrm{~d} r \mathrm{~d} z}_{=0}+\mathrm{BT}
\end{aligned}
$$

In Eq. (B18), BT is a boundary term arising during the integration by parts, whose value is non-zero owing to the existence of the control velocity. The detailed calculation of the boundary term can be found in Ref. 53. For a jet located at the pipe wall, one ultimately finds that

$$
\begin{aligned}
& \int_{\Sigma} \hat{\boldsymbol{q}}^{\dagger} \cdot \boldsymbol{F}_{(2)} r \mathrm{~d} r \mathrm{~d} z \\
& \quad=-\int_{\partial \Sigma_{\mathrm{w}}}\left(\hat{p}^{\dagger}+\frac{1}{R e}\left(\left(\nabla \hat{\boldsymbol{u}}^{\dagger}+\nabla \hat{\boldsymbol{u}}^{\dagger}\right) \cdot \boldsymbol{n}\right) \cdot \boldsymbol{n}\right) U_{\mathrm{c}}^{\perp} r \mathrm{~d} l
\end{aligned}
$$

thus yielding an amplitude equation

$$
\frac{\mathrm{d} A}{\mathrm{~d} T}=\alpha A^{2}+\beta \Delta+\Gamma
$$

with

$$
\Gamma=\frac{1}{\mathcal{S}} \int_{\partial \Sigma_{\mathrm{w}}}\left(\hat{p}^{\dagger}+\frac{1}{\operatorname{Re}}\left(\left(\nabla \hat{\boldsymbol{u}}^{\dagger}+\nabla \hat{\boldsymbol{u}}^{\dagger^{T}}\right) \cdot \boldsymbol{n}\right) \cdot \boldsymbol{n}\right) U_{\mathrm{c}}^{\perp} r \mathrm{~d} l .
$$

A similar equation is obtained for a modification of the inlet velocity profile, the only difference being that the boundary term is computed by integration along the inlet section. Equation (B20) is ultimately recast in terms of the physical amplitude $a=\epsilon A$ according to

$$
\frac{\mathrm{d} a}{\mathrm{~d} t}=\alpha a^{2}+\beta\left(S_{*}-S\right)+\gamma,
$$

with

$$
\gamma=\epsilon^{2} \Gamma=\frac{1}{\mathcal{S}} \int_{\partial \Sigma_{\mathrm{w}}}\left(\hat{p}^{\dagger}+\frac{1}{R e}\left(\left(\nabla \hat{\boldsymbol{u}}^{\dagger}+\nabla \hat{\boldsymbol{u}}^{\dagger^{T}}\right) \cdot \boldsymbol{n}\right) \cdot \boldsymbol{n}\right) u_{\mathrm{c}}^{\perp} r \mathrm{~d} l .
$$

${ }^{1}$ N. C. Lambourne and D. W. Bryer, "The Bursting of leading-edge vortices-Some observations and discussion of the phenomenon," Aero. Res. Counc. 3282, 1 (1961).

${ }^{2}$ P. Billant, J.-M. Chomaz, and P. Huerre, "Experimental study of vortex breakdown in swirling jets," J. Fluid Mech. 376, 183 (1998).

${ }^{3}$ T. Sarpkaya, "On stationary and travelling vortex breakdown," J. Fluid Mech. 45, 545 (1971).

${ }^{4}$ T. Benjamin, "Theory of the vortex breakdown phenomenon," J. Fluid Mech. 14, 593 (1962).

${ }^{5}$ M. Hall, "Vortex breakdown," Annu. Rev. Fluid Mech. 4, 195 (1972).

${ }^{6}$ S. Leibovich, "The structure of vortex breakdown," Annu. Rev. Fluid Mech. 10, 221 (1978).

${ }^{7}$ M. Escudier, J. Bornstein, and T. Maxworthy, "The dynamics of confined vortices," Proc. R. Soc. Lond. A 382, 335 (1982).

${ }^{8}$ M. Escudier, "Vortex breakdown: Observations and explanations," Prog. Aerosp. Sci. 25, 189 (1988).

${ }^{9}$ A. Mitchell and J. Delery, "Research into vortex breakdown control," Prog. Aerosp. Sci. 37, 385 (2001).

${ }^{10}$ M. R. Ruith, P. Chen, E. Meiburg, and T. Maxworthy, "Three-dimensional vortex breakdown in swirling jets and wakes: Direct numerical simulation," J. Fluid Mech. 486, 331 (2003).

${ }^{11}$ I. Delbende, J.-M. Chomaz, and P. Huerre, "Absolute/convective instabilities in the Batchelor vortex: A numerical study of the linear impulse response," J. Fluid Mech. 355, 229 (1998).

${ }^{12} \mathrm{D}$. Lim and L. Redekopp, "Absolute instability conditions for variable density, swirling jet flows,” Eur. J. Mech. B/Fluids 17, 165 (1998).

${ }^{13}$ T. Loiseleux, J.-M. Chomaz, and P. Huerre, "The effect of swirl on jets and wakes: Linear instability of the Rankine vortex with axial flow," Phys. Fluids 10, 1120 (1998).

${ }^{14}$ C. Olendraru, A. Sellier, and P. Huerre, "Inviscid instability of the Batchelor vortex: Absolute/convective transition and spatial branches," Phys. Fluids 11, 1805 (1999).

${ }^{15}$ F. Gallaire and J.-M. Chomaz "Mode selection in swirling jet experiments: A linear stability analysis," J. Fluid Mech. 494, 223 (2003).

${ }^{16}$ F. Gallaire, M. Ruith, E. Meiburg, J.-M. Chomaz, and P. Huerre "Spiral vortex breakdown as a global mode," J. Fluid Mech. 549, 71 (2006).

${ }^{17}$ G. L. Brown and J. M. Lopez, "Axisymmetric vortex breakdown. II. Physical mechanisms,” J. Fluid Mech. 221, 553 (1990).

${ }^{18}$ P. S. Beran and F. E. C. Culick, "The role of non-uniqueness in the development of vortex breakdown in tubes," J. Fluid Mech. 242, 491 (1992).

${ }^{19} \mathrm{P}$. S. Beran, "The time-asymptotic behavior of vortex breakdown in tubes," Comp. Fluids 23, 913 (1994).

${ }^{20} \mathrm{~J}$. M. Lopez, "On the bifurcation structure of axisymmetric vortex breakdown in a constricted pipe," Phys. Fluids 6, 3683 (1994).

${ }^{21}$ E. Viazmyna, J. W. Nichols, J.-M. Chomaz, and P. J. Schmid, “The bifurcation structure of viscous steady axisymmetric vortex breakdown with open lateral boundaries," Phys. Fluids 21, 074107 (2009).

${ }^{22}$ H. B. Squire, "Analysis of the vortex breakdown phenomenon," Miszallaneen der Angewandten Mechanik (Akademie Verlag, Berlin, 1960), p. 306.

${ }^{23}$ S. Wang and Z. Rusak, "On the stability of an axisymmetric rotating flow in a pipe," Phys. Fluids 8, 1007 (1996).

${ }^{24}$ S. Wang and Z. Rusak, "On the stability of non-columnar swirling flows," Phys. Fluids 8, 1017 (1996).

${ }^{25} \mathrm{~S}$. Wang and Z. Rusak, "The dynamics of a swirling flow in a pipe and transition to axisymmetric vortex breakdown,” J. Fluid Mech. 340, 177 (1997).

${ }^{26}$ Z. Rusak, K. P. Judd, and S. Wang, "The effect of small pipe divergence on near-critical swirling flows," Phys. Fluids 9, 2273 (1997).

${ }^{27}$ S. Wang and Z. Rusak, "The effect of slight viscosity on a near-critical swirling flow in a pipe," Phys. Fluids 9, 1914 (1997).

${ }^{28}$ Z. Rusak, K. P. Judd, and S. Wang, "The stability of noncolumnar swirling flows in diverging streamtubes," Phys. Fluids 13, 2835 (2001).

${ }^{29}$ Z. Rusak and C. C. Meder, "Near-critical swirling flow in a slightly contracting pipe," AIAA J. 42, 2284 (2004).

${ }^{30}$ T. W. Mattner, P. N. Joubert, and M. S. Chong, "Vortical flow. Part 1. Flow through a constant-diameter pipe," J. Fluid Mech. 463, 259 (2002).

${ }^{31}$ F. Gallaire, J.-M. Chomaz, and P. Huerre, "Closed-loop control of vortex breakdown: A model study,” J. Fluid Mech. 511, 67 (2004).

${ }^{32}$ D. Darmofal, "Comparisons of experimental and numerical results for axisymmetric vortex breakdown in pipes," Comp. Fluids 25, 353 (1996).

${ }^{33}$ A. K. Garg and S. Leibovich, "Spectral characteristics of vortex breakdown flow-fields," Phys. Fluids 22, 2053 (1979).

${ }^{34}$ P. Meliga, J.-M. Chomaz, and D. Sipp, "Global mode interaction and pattern selection in the wake of a disk: A weakly nonlinear expansion," J. Fluid Mech. 633, 159 (2009). 
${ }^{35}$ J. Matsumoto and M. Kawahara, "Shape identification for fluid-structure interaction problem using improved bubble element," Int. J. Comp. Fluid Dyn. 15, 33 (2001).

${ }^{36}$ D. Barkley, M. G. M. Gomes, and R. D. Henderson, "Three-dimensional instability in flow over a backward-facing step," J. Fluid Mech. 473, 167 (2002).

${ }^{37}$ T. A. Davis and I. S. Duff, "An unsymmetric-pattern multifrontal method for sparse LU factorization," SIAM J. Matrix Anal. Appl. 18, 140 (1997).

${ }^{38}$ T. A. Davis, "A column pre-ordering strategy for the unsymmetric-pattern multifrontal method," ACM Trans. Math. Softw. 30, 165 (2004).

${ }^{39}$ H. B. Keller, "Numerical solutions of bifurcation and nonlinear eigenvalue problems," Applications of Bifurcation Theory, edited by P. Rabinowitz (Academic, New York, 1977), p. 359.

${ }^{40}$ U. Ehrenstein and F. Gallaire, "On two-dimensional temporal modes in spatially evolving open flows: The flat-plate boundary layer," J. Fluid Mech. 536, 209 (2005).

${ }^{41}$ M. R. Ruith, P. Chen, and E. Meiburg, "Development of boundary conditions for direct numerical simulations of three-dimensional vortex breakdown phenomena in semi-infinite domains," Comp. Fluids 33, 1225 (2004).

${ }^{42}$ K. O. Friedrichs, Spectral Theory of Operators in Hilbert Space (SpringerVerlag, New York, 1973).

${ }^{43}$ O. Marquet, M. Lombardi, J.-M. Chomaz, D. Sipp, and L. Jacquin, "Direct and adjoint global modes of a recirculation bubble: Lift-up and convective non-normalities," J. Fluid Mech. 622, 1 (2009).

${ }^{44}$ D. C. Hill, NASA Technical Report No. 103858, 1992.

${ }^{45}$ O. Marquet, D. Sipp, and L. Jacquin, "Sensitivity analysis and passive control of cylinder flow," J. Fluid Mech. 615, 221 (2008).

${ }^{46} \mathrm{P}$. Meliga and J.-M. Chomaz, "Global modes in a confined impinging jet: Application to heat transfer and control," Theor. Comput. Fluid Dyn. 25, 179 (2011).
${ }^{47}$ P. Meliga, D. Sipp, and J.-M. Chomaz, “Open-loop control of compressible afterbody flows using adjoint methods," Phys. Fluids 22, 054109 (2010).

${ }^{48}$ J. M. Lopez, Y. D. Cui, F. Marques, and T. T. Lim, "Quenching of vortex breakdown oscillations via harmonic modulation,” J. Fluid Mech. 599, 441 (2008).

${ }^{49}$ Y. D. Cui, J. M. Lopez, T. T. Lim, and F. Marques, "Harmonically forced enclosed swirling flow," Phys. Fluids 21, 034106 (2009).

${ }^{50} \mathrm{Z}$. Rusak, "The interaction of near-critical swirling flows in a pipe with inlet azimuthal vorticity perturbations," Phys. Fluids 10, 1672 (1998).

${ }^{51}$ B. Leclaire and D. Sipp, "A sensitivity study of vortex breakdown onset to upstream boundary conditions,” J. Fluid Mech. 645, 81 (2009).

${ }^{52}$ P. J. Schmid and D. S. Henningson, Stability and Transition in Shear Flows (Springer-Verlag, New York, 2001).

${ }^{53}$ F. Giannetti and P. Luchini, "Structural sensitivity of the first instability of the cylinder wake," J. Fluid Mech. 581, 167 (2007).

${ }^{54}$ F. Hecht, FreeFem ++ software, Jacques-Louis Lions laboratory (CNRSUniversité Pierre and Marie Curie), Paris, France, 2011. See http:// www.freefem.org.

${ }^{55}$ Relation (39) relies on the assumption that a uniform modification of the inlet velocity acts by rescaling the swirl. This is not rigorously true as such a modification also acts by rescaling the Reynolds number. Additional numerical simulations have therefore been run by increasing the dimensional inlet velocity from $w_{0}$ to $(1+\eta) w_{0}$, which show that all results obtained keeping the Reynolds number constant are identical to the value $1.893(1+\eta)$ issuing from a complete dynamic similarity hypothesis. In other words, viscosity has almost no effect in the range of flow rates considered and a uniform modification of the inlet velocity acts through the only rescaling of the swirl. 